\title{
High-scale validity of a two-Higgs doublet scenario: a study including LHC data
}

\section{Nabarun Chakrabarty, Ujjal Kumar Dey and Biswarup Mukhopadhyaya}

Regional Centre for Accelerator-based Particle Physics, Harish-Chandra Research Institute, Chhatnag Road, Jhunsi, Allahabad 211 019, India

E-mail: nabarunc@hri.res.in, ujjaldey@hri.res.in, biswarup@hri.res.in

ABSTRACT: We consider the conditions for the validity of a two-Higgs doublet model at high energy scales, together with all other low- and high-energy constraints. The constraints on the parameter space at low energy, including the measured value of the Higgs mass and the signal strengths in channels are juxtaposed with the conditions of vacuum stability, perturbativity and unitarity at various scales. We find that a scenario with an exact $\mathbb{Z}_{2}$ symmetry in the potential cannot be valid beyond about $10 \mathrm{TeV}$ without the intervention of additional physics. On the other hand, when the $\mathbb{Z}_{2}$ symmetry is broken, the theory can be valid even up to the Planck scale without any new physics coming in. The interesting feature we point out is that such high-scale validity is irrespective of the uncertainty in the top quark mass as well as $\alpha_{s}\left(M_{Z}\right)$, in contrast with the standard model with a single Higgs doublet. It is also shown that the presence of a CP-violating phase is allowed when the $\mathbb{Z}_{2}$ symmetry is relaxed. The allowed regions in the parameter space are presented for each case. The results are illustrated in the context of a Type-II scenario.

Keywords: Beyond Standard Model, Higgs Physics

ARXIV EPRINT: 1407.2145 


\section{Contents}

1 Introduction 1

2 The two-Higgs-doublet scenario and the scalar potential: basic features 3

3 Theoretical and experimental constraints 5

$\begin{array}{ll}3.1 \text { Perturbativity, unitarity and vacuum stability } & 6\end{array}$

$\begin{array}{lll}3.2 & \text { Higgs mass constraints } & 7\end{array}$

$\begin{array}{ll}3.3 & \text { Oblique parameter constraints }\end{array}$

3.4 Collider constraints 8

4 Results with exact discrete symmetry 9

5 Results with softly broken discrete symmetry 12

6 Results with quartic terms breaking the discrete symmetry 18

$\begin{array}{lll}7 & \text { Summary and conclusions } & 22\end{array}$

A Renormalization group (RG) equations $\quad 23$

B Unitarity bounds $\quad 24$

\section{Introduction}

The Higgs sector of the standard electroweak model (SM) continues to appear enigmatic from several angles. The existence of such a sector, comprising at least one scalar doublet, and driving the spontaneous symmetry breakdown $\mathrm{SU}(2)_{L} \times \mathrm{U}(1)_{Y} \longrightarrow \mathrm{U}(1)_{\mathrm{EM}}$ is almost impossible to deny now. It is also widely agreed that the Large Hadron Collider (LHC) has found $[1,2]$ a neutral boson with mass around $125 \mathrm{GeV}$, which is almost certainly of spin zero [3] and dominantly a CP-even field [4-7]. However, despite the properties of the boson being consistent with that of the SM Higgs, rather persistent enquiries are on, to find out whether the electroweak symmetry breaking sector also contains some signature of physics beyond the standard model. The LHC data till date leaves room for such new physics.

Two sets of standpoints are noticed in such enquiries. First of all, with spin-1/2 fermions showing family replication, it is not obvious why the part of the matter sector containing spin-zero particles should also not have similar repetition. With this in view, multi-doublet scenarios are under regular scrutiny, the most widely investigated models being those with two Higgs doublets [8, 9]. An extended electroweak symmetry breaking sector entails a rich phenomenology, including additional sources of $\mathrm{CP}$ violation [10]. Of course, scalars belonging to higher representations of $\mathrm{SU}(2)$ have also attracted attention, especially triplets which can play a role in the so-called Type-II mechanism of neutrino mass 
generation [11]. Secondly, even with just one doublet (leading to a single physical scalar), the Higgs mass is not stable under quadratically divergent radiative corrections, and it is somewhat artificial (or 'fine-tuned') to have a $125 \mathrm{GeV}$ Higgs if the cut-off for the SM is much higher than a $\mathrm{TeV}$ or so. Furthermore, it is also not clear that the SM scalar potential retains a finite and stable minimum at high scales. But for the yet uncertain measurement of the top quark mass, which is crucial in governing the evolution of the Higgs self-coupling via Yukawa interactions, we may be doomed to live in an unstable or metastable vacuum if no new physics intervenes within the scale $10^{8-10} \mathrm{GeV}[12-16]$. Therefore, the ultraviolet incompleteness of the current scheme of electroweak symmetry breaking looms up as a distinct possibility, even if one disregards the somewhat philosophical issue of naturalness.

In this paper, we follow these two standpoints in tandem. We take up a two-Higgs doublet scenario as the minimal extension of the standard electroweak theory, assessing its viability as well as sufficiency modulo all available constraints. The motivation for the study is that the proportionality constant between the top quark mass and its coupling to the $125 \mathrm{GeV}$ scalar is different from its SM value when more than one doublet is taken. Consequently, the dependence of the vacuum stability limit on the top quark mass is expected to be different. However, one can make precise and quantitative statements on the matter only when one takes cognizance of the exact scenario, and includes the complete set of renormalisation group equations appropriate for it. This is precisely what we aim to do here, using a two-Higgs doublet scenario at various levels of generality.

The desired suppression of flavour-changing Yukawa interactions is best implemented by imposing a discrete symmetry on such models, thus preventing both the doublets from coupling with $T_{3}=+1 / 2$ and $-1 / 2$ fermions simultaneously. It is possible to go beyond such imposition and examine two Higgs doublets in a 'basis-independent' formulation [17-19]. However, we feel that our central issue, namely, the evolution of the Higgs selfinteraction(s), is amenable to a more transparent study if one adheres to a specific Yukawa scheme. With this in view, we adopt the so-called Type-II scenario for our study [8], to illustrate our point.

We begin by examining the situation when the discrete symmetry is exact, and derive the constraints on the low-energy values of the parameters of this scenario. The lighter neutral scalar mass being around $125 \mathrm{GeV}$ is of course the prime requirement here, and constraints from rare processes such as $b \rightarrow s \gamma$ are also included. In addition, the constraints from perturbativity of all scalar quartic couplings are considered, together with those from vacuum stability. The parameter space thus validated is further examined in the light of the perturbativity and vacuum stability conditions at high scales. Thus we identify the parameter regions that keep a two-Higgs doublet scenario valid up to different levels of high scales- an exercise that reveals rather severe limits. The same investigation is carried out for cases where the discrete symmetry is broken by soft (dimension-2) and hard (dimension-4) terms in turn, with the Yukawa coupling assignment remaining (for simplicity) the same as in the case with unbroken symmetry. The effect of a CP-violating phase is also demonstrated. Finally, the regions found to be allowed from all the above considerations, at both low- and high-scales, are pitted against the existing data from the LHC in different channels. Thus we identify parameter regions that are consistent with the 
measured signal strengths in different channels. This entire study is aimed at indicating how far a two-Higgs doublet model can remain valid, not only at the LHC energy but also up to various high scales without further intervention of new physics.

Although a number of recent studies have addressed some similar questions [20-22], the present study has gone beyond them on the following points:

- Our study reveals that the high scale validity of the theory is less sensitive to the precise value of the top quark mass than in the SM. Regions in the parameter space are identified, for which the theory has no cut-off till the Planck scale, even though the top quark mass can be at the upper edge of the allowed band. Similarly, the high scale validity of the model is insensitive to $\alpha_{s}\left(M_{Z}\right)$.

- We find that it is rather difficult to retain the validity of a two-Higgs doublet scenario well above a $\mathrm{TeV}$ with the discrete symmetry intact. Also, large values of $\tan \beta$, the ratio of the vacuum expectation values (vev) of the two doublets, are mostly disfavoured in this case.

- With the discrete symmetry broken, the theory can circumvent ultraviolet cut-offs. There is a correlation between allowed $\tan \beta$ and the extent of symmetry breaking, when it comes to validity up to the Planck scale.

- We examine the constraints on the model including a CP-violating phase [23-26]. In fact, since the existence of a phase is a natural consequence of relaxing the discrete symmetry, the high-scale validity of a two Higgs doublet model may be argued to be contingent on the possibility of CP-violation in the scalar potential.

- We have performed a detailed examination of the validity of the scenario at both low- and high scales, including dimension-4 discrete symmetry breaking terms in our analysis. The LHC constraints are also imposed in this situation.

We remind the reader of the broad features of a two-Higgs doublet scenario in section 2. In section 3, we list and explain all the constraints that the scenario is subjected to, at both the low and high scales. Sections 4, 5 and 6 contain, in turn, the results of our analysis, with the discrete symmetry intact, softly broken and broken by hard terms, respectively. We summarise and conclude in section 7 .

\section{The two-Higgs-doublet scenario and the scalar potential: basic features}

In the present work, we consider the most general renormalisable scalar potential for two doublets $\Phi_{1}$ and $\Phi_{2}$, each having hypercharge $(+1)$,

$$
\begin{aligned}
V\left(\Phi_{1}, \Phi_{2}\right)= & m_{11}^{2} \Phi_{1}^{\dagger} \Phi_{1}+m_{22}^{2} \Phi_{2}^{\dagger} \Phi_{2}-m_{12}^{2}\left(\Phi_{1}^{\dagger} \Phi_{2}+\Phi_{2}^{\dagger} \Phi_{1}\right)+\frac{\lambda_{1}}{2}\left(\Phi_{1}^{\dagger} \Phi_{1}\right)^{2}+\frac{\lambda_{2}}{2}\left(\Phi_{2}^{\dagger} \Phi_{2}\right)^{2} \\
& +\lambda_{3} \Phi_{1}^{\dagger} \Phi_{1} \Phi_{2}^{\dagger} \Phi_{2}+\lambda_{4} \Phi_{1}^{\dagger} \Phi_{2} \Phi_{2}^{\dagger} \Phi_{1}+\frac{\lambda_{5}}{2}\left[\left(\Phi_{1}^{\dagger} \Phi_{2}\right)^{2}+\left(\Phi_{2}^{\dagger} \Phi_{1}\right)^{2}\right] \\
& +\lambda_{6} \Phi_{1}^{\dagger} \Phi_{1}\left(\Phi_{1}^{\dagger} \Phi_{2}+\Phi_{2}^{\dagger} \Phi_{1}\right)+\lambda_{7} \Phi_{2}^{\dagger} \Phi_{2}\left(\Phi_{1}^{\dagger} \Phi_{2}+\Phi_{2}^{\dagger} \Phi_{1}\right) .
\end{aligned}
$$


The parameters $m_{12}, \lambda_{5}, \lambda_{6}$ and $\lambda_{7}$ could be complex in general, although the phase in one of them can be removed by redefinition of of the relative phase between $\Phi_{1}$ and $\Phi_{2}$. Thus this scenario in general has the possibility of CP-violation in the scalar sector.

In a general two-Higgs-doublet model (2HDM), a particular fermion can couple to both $\Phi_{1}$ and $\Phi_{2}$. However this would lead to the flavor changing neutral currents (FCNC) at the tree level [27-30]. ${ }^{1}$ One way to avoid such FCNC is to impose a $\mathbb{Z}_{2}$ symmetry, such as one that demands invariance under $\Phi_{1} \rightarrow-\Phi_{1}$ and $\Phi_{2} \rightarrow \Phi_{2}$. This type of symmetry puts restrictions on the scalar potential. The $\mathbb{Z}_{2}$ symmetry is exact as long as $m_{12}, \lambda_{6}$ and $\lambda_{7}$ vanish, when the scalar sector also becomes CP-conserving. The symmetry is said to be broken softly if it is violated in the quadratic terms only, i.e., in the limit where $\lambda_{6}$ and $\lambda_{7}$ vanish but $m_{12}$ does not. Finally, a hard breaking of the $\mathbb{Z}_{2}$ symmetry is realized when it is broken by the quartic terms as well. Thus in this case, $m_{12}, \lambda_{6}$ and $\lambda_{7}$ all are non-vanishing in general.

As mentioned in the introduction, we focus on a specific scheme of coupling fermions to the doublets. This scheme is referred to in the literature as the Type-II $2 \mathrm{HDM}$, where the down type quarks and the charged leptons couple to $\Phi_{1}$ and the up type quarks, to $\Phi_{2}$ [33]. This can be ensured through the discrete symmetry $\Phi_{1} \rightarrow-\Phi_{1}$ and $\psi_{R}^{i} \rightarrow-\psi_{R}^{i}$, where $\psi$ is charged leptons or down type quarks and $i$ represents the generation index. Although we start by analysing the high-scale validity of the model with $m_{12}=\lambda_{6}=\lambda_{7}=0$, we subsequently include the effects of both soft and hard breaking of $\mathbb{Z}_{2}$ in turn, which bring back these parameters. The two simplifications that we still make are as follows: (a) the phases of $\lambda_{6}$ and $\lambda_{7}$ are neglected though that of $m_{12}$ is considered, and (b) the Yukawa coupling assignments of $\Phi_{1}$ and $\Phi_{2}$ are left unchanged.

Minimization of the scalar potential in eq. (2.1) yields

$$
\left\langle\Phi_{1}\right\rangle=\left(\begin{array}{c}
0 \\
\frac{v_{1}}{\sqrt{2}}
\end{array}\right), \quad\left\langle\Phi_{2}\right\rangle=\left(\begin{array}{c}
0 \\
\frac{v_{2}}{\sqrt{2}}
\end{array}\right),
$$

where the vacuum expectation values (vev) are often expressed in terms of the $M_{Z}$ and the ratio

$$
\tan \beta=\frac{v_{2}}{v_{1}} .
$$

We parametrise the doublets in the following fashion,

$$
\Phi_{i}=\frac{1}{\sqrt{2}}\left(\begin{array}{c}
\sqrt{2} w_{i}^{+} \\
v_{i}+h_{i}+i z_{i}
\end{array}\right) \text { for } i=1,2 .
$$

Since the basis used in $V\left(\Phi_{1}, \Phi_{2}\right)$ allows mixing between the two doublets, one diagonalises the charged and neutral scalar mass matrices to obtain the physical states. There are altogether eight mass eigenstates, three of which become the longitudinal components of the $W^{ \pm}$and $Z$ gauge bosons. Of the remaining five, there is a mutually conjugate pair of charged scalars $\left(H^{ \pm}\right)$, two neutral scalars $(H, h)$ and a neutral pseudoscalar $(A)$, when

\footnotetext{
${ }^{1}$ In context of a typical flavour changing scenario, it has been shown in [31, 32] that the FCNCs are stable under RG evolution to a fairly large degree.
} 
there is no CP-violation. Otherwise, a further mixing occurs between $(H, h)$ and $A$. The compositions of the mass eigenstates $H$ and $h$ depend on the mixing angle $\alpha$.

In the absence of CP-violation, the squared masses of these physical scalars and the mixing angle $\alpha$ can be expressed as [34],

$$
\begin{aligned}
m_{A}^{2} & =\frac{m_{12}^{2}}{s_{\beta} c_{\beta}}-\frac{1}{2} v^{2}\left(2 \lambda_{5}+\frac{\lambda_{6}}{t_{\beta}}+\lambda_{7} t_{\beta}\right), \\
m_{H^{ \pm}}^{2} & =m_{A}^{2}+\frac{1}{2} v^{2}\left(\lambda_{5}-\lambda_{4}\right), \\
m_{h}^{2} & =\frac{1}{2}\left[(A+B)-\sqrt{(A-B)^{2}+4 C^{2}}\right], \\
m_{H}^{2} & =\frac{1}{2}\left[(A+B)+\sqrt{(A-B)^{2}+4 C^{2}}\right], \\
\tan 2 \alpha & =\frac{2 C}{A-B},
\end{aligned}
$$

where we have defined,

$$
\begin{aligned}
& A=m_{A}^{2} s_{\beta}^{2}+v^{2}\left(\lambda_{1} c_{\beta}^{2}+\lambda_{5} s_{\beta}^{2}+2 \lambda_{6} s_{\beta} c_{\beta}\right), \\
& B=m_{A}^{2} c_{\beta}^{2}+v^{2}\left(\lambda_{2} s_{\beta}^{2}+\lambda_{5} c_{\beta}^{2}+2 \lambda_{7} s_{\beta} c_{\beta}\right), \\
& C=-m_{A}^{2} s_{\beta} c_{\beta}+v^{2}\left[\left(\lambda_{3}+\lambda_{4}\right) s_{\beta} c_{\beta}+\lambda_{6} c_{\beta}^{2}+\lambda_{7} s_{\beta}^{2}\right] .
\end{aligned}
$$

Furthermore, the interactions of the various charged and neutral scalars to the up- and down-type fermions are functions of $\alpha$ and $\beta$. Their detailed forms in different 2HDM scenarios, including the Type-II model adopted here for illustration, can be found in the literature [9].

\section{$3 \quad$ Theoretical and experimental constraints}

Next, we subject the Type-II 2HDM using various theoretical and experimental constraints (though the most binding ones are often irrespective of the specific type of $2 \mathrm{HDM}$ ). It should be remembered at the outset that the most general $\mathbb{Z}_{2}$ violating $2 \mathrm{HDM}$ has seven quartic couplings, namely, $\lambda_{i}(i=1, \ldots, 7)$, in addition to $\tan \beta$ and $m_{12}$, totalling to nine free parameters. Though such a nine-dimensional parameter is prima facie large enough to accommodate any phenomenology, the set of constraints under consideration below can ultimately become quite restrictive.

We discuss the theoretical constraints in subsection 3.1, and take up the experimental/phenomenological ones in the subsequent subsections. It should be noted that the parameter space is being constrained in two distinct ways. Subsections 3.2-3.4 list essentially low-energy constraints which apply at the energy scale of the subprocesses leading to Higgs production. The various masses and couplings get restricted by the requirement of satisfying them. However, while such a strategy is valid for the discussion of subsection 3.1 as well, we additionally require the conditions laid down there to hold at various high scales, too. This not only restricts the low-energy parameters more severely, but also answers the main question asked in this paper, namely, to what extent the $2 \mathrm{HDM}$ can be deemed 'ultraviolet complete'. 


\subsection{Perturbativity, unitarity and vacuum stability}

For the 2HDM to behave as a perturbative quantum field theory at any given scale, one must impose the conditions $\left|\lambda_{i}\right| \leq 4 \pi(i=1, \ldots, 7)$ and $\left|y_{i}\right| \leq \sqrt{4 \pi}(i=t, b, \tau)$ at that scale. $^{2}$ On applying such conditions, one implies upper bounds on the values of the couplings at low as well as high scales.

Next, we impose the more stringent condition of unitarity on the tree-level scattering amplitudes involving the scalar degrees of freedom. In a model with an extended scalar sector, the scattering amplitudes are taken between various two-particle states constituted out of the fields $w_{i}^{ \pm}, h_{i}$ and $z_{i}$ corresponding to the parametrisation of eq. (2.4). Maintaining this, there will be neutral two-particle states (e.g., $w_{i}^{+} w_{j}^{-}, h_{i} h_{j}, z_{i} z_{j}, h_{i} z_{j}$ ) as well as singly charged two-particle states (e.g., $w_{i}^{ \pm} h_{j}, w_{i}^{ \pm} z_{j}$ ). The various two particle initial and final states give rise to a $2 \rightarrow 2$ scattering matrix whose elements are the lowest order partial wave expansion coefficients in the corresponding amplitudes. The method used by Lee, Quigg and Thacker (LQT) [35] prompts us to consider the eigenvalues of this two-particle scattering matrix $[21,36,37]$. These eigenvalues, labelled as $a_{i}$, should satisfy the condition $\left|a_{i}\right| \leq 8 \pi$. Again, these conditions apply to high scales as well, if we expect perturbativity to hold.

When the quartic part of the scalar potential preserves CP [38, 39] and $\mathbb{Z}_{2}$ symmetries, the LQT eigenvalues are discussed in [40-42]. For $\lambda_{6}, \lambda_{7}=0$, we follow the procedure and notation of [40] and [42]. However, the matrices for coupled-channel analysis including $\lambda_{6}$ and $\lambda_{7}$ are derived by us (see $\mathcal{M}_{\mathrm{NC}}$ and $\mathcal{M}_{\mathrm{CC}}$ in appendix B). The general formulae including $\lambda_{6}, \lambda_{7}$, are given in appendix B.

The condition to be taken up next is that of vacuum stability. For the scalar potential of a theory to be stable, it must be bounded from below in all directions. This condition is threatened if the quartic part of the scalar potential, which is responsible for its behaviour at large field values, turns negative. Avoiding such a possibility up to any given scale ensures vacuum stability up to that scale. The issue of vacuum stability in context of a 2HDM has been discussed in detail in [20, 22, 43-45].

The 2HDM potential has eight real scalar fields. By studying the behaviour of the quartic part of its scalar potential along different field directions, one arrives at the following conditions $[9,46]$,

$$
\begin{array}{ll}
\operatorname{vsc} 1: & \lambda_{1}>0 \\
\operatorname{vsc} 2: & \lambda_{2}>0 \\
\operatorname{vsc} 3: & \lambda_{3}+\sqrt{\lambda_{1} \lambda_{2}}>0 \\
\operatorname{vsc} 4: & \lambda_{3}+\lambda_{4}-\left|\lambda_{5}\right|+\sqrt{\lambda_{1} \lambda_{2}}>0 \\
\operatorname{vsc} 5: & \frac{1}{2}\left(\lambda_{1}+\lambda_{2}\right)+\lambda_{3}+\lambda_{4}+\lambda_{5}-2\left|\lambda_{6}+\lambda_{7}\right|>0 .
\end{array}
$$

\footnotetext{
${ }^{2}$ The conditions are slightly different for the two types of couplings. The reason becomes clear if we note that the perturbative expansion parameter for $2 \rightarrow 2$ processes driven by the quartic couplings is $\lambda_{i}$. The corresponding parameter for Yukawa-driven scattering processes is $\left|y_{i}\right|^{2}$.
} 
The reader is reminded that the above conditions indicate a stable electroweak vacuum and not a metastable one. The couplings in the general $\mathbb{Z}_{2}$ violating Type-II $2 \mathrm{HDM}$ evolve from a low scale to a high scale according to a set of renormalisation group (RG) equations listed in the appendix A. If one proposes the UV cut-off scale of the model to be some $\Lambda_{\mathrm{UV}}$, it might so happen that the couplings grow with the energy scale and hit the Landau pole before $\Lambda_{\mathrm{UV}}$. A second, still unacceptable, possibility is that of the LQT eigenvalues crossing their unitarity limits. The RG evolution of the $2 \mathrm{HDM}$ couplings has been recently studied in $[47,48]$. Finally, the stability conditions can get violated below $\Lambda_{\mathrm{UV}}$, making the scalar potential unbounded from below. All these problems are avoided if one postulates that all of the conditions laid down above are valid up to $\Lambda_{\mathrm{UV}}$, which marks the maximum energy up to which the $2 \mathrm{HDM}$ can be valid without the intervention of any additional physics.

\subsection{Higgs mass constraints}

The spectrum of a generic $2 \mathrm{HDM}$ consists of a charged scalar, a CP-odd neutral scalar and two CP-even neutral scalars. Since the LHC has observed a CP-even neutral boson around $125 \mathrm{GeV}$, we allow only those regions in the parameter space for which $h$, the lighter neutral scalar, lies in the mass range $124.53-126.18 \mathrm{GeV}$ which is within $2 \sigma$ error limits following [49]. In addition, the charged scalar is required to have a mass greater than $315 \mathrm{GeV}$ due to low energy constraints, coming mainly from $b \rightarrow s \gamma[50,51]$. The benchmark points used by us are also consistent with $B \rightarrow \tau \nu_{\tau}, B_{s} \rightarrow \mu^{+} \mu^{-}$and $B^{0}-\overline{B^{0}}$ mixing $[51,52]$.

\subsection{Oblique parameter constraints}

The presence of an additional $\mathrm{SU}(2)$ doublet having a hypercharge $Y=1$ modifies the electroweak oblique parameters [53]. It is to be noted that since the couplings of the fermions to gauge bosons remain unaltered even after the introduction of the second doublet, all the additional contributions come from the scalar sector of the 2HDM. The oblique parameters can be decomposed as,

$$
\begin{aligned}
& S=S_{\mathrm{SM}}+\Delta S \\
& T=T_{\mathrm{SM}}+\Delta T,
\end{aligned}
$$

where $S_{\mathrm{SM}}$ and $T_{\mathrm{SM}}$ denote the Standard Model (SM) contributions and $\Delta S$ and $\Delta T$ denote any new physics effect. The central value is the contribution coming from the standard model with the reference values $m_{h, \text { ref }}=125.0 \mathrm{GeV}$ and $M_{t, \text { ref }}=173.1 \mathrm{GeV}$ where $M_{t}$ denotes the pole mass of the top quark. The expressions for $\Delta S$ and $\Delta T$ for a general 2HDM can be found in [19, 54-56]. The corresponding bounds we have used are $|\Delta S|<0.11$ and $|\Delta T|<0.13$ [57]. The splitting amongst the scalar masses affects the $T$ parameter, which is linked to the custodial $\mathrm{SU}(2)$ symmetry. Typically for $m_{12}=0, T$ prevents large mutual splitting among states other than the lightest neutral scalar. For $m_{12} \neq 0$, the scalars other than the light neutral one have masses $\sim m_{12}$. As $m_{12}$ is increased, the masses approach the decoupling limit, and in that case, the oblique electroweak constraints are naturally satisfied, as the $2 \mathrm{HDM}$ approaches the SM in that case. The consistency with 
these parameters has nevertheless been explicitly ensured at each allowed point of the parameter space.

\subsection{Collider constraints}

Apart from the theoretical constraints discussed above, we also strive to find the region of parameter space of a $2 \mathrm{HDM}$ allowed by the recent Higgs data. The ATLAS [58-60] and CMS [61] collaborations have measured the production cross section for a $\sim 125 \mathrm{GeV}$ Higgs multiplied by its branching ratios to various possible channels. In our case, since the underlying theory is a $2 \mathrm{HDM}$, all the cross sections and decay widths get modified compared to the corresponding SM values. For example, the production cross section of the light neutral Higgs through gluon fusion will get rescaled in the case of a 2HDM due to the fact that the fermionic couplings of the $125 \mathrm{GeV}$ Higgs are now changed with respect to the SM values by appropriate multiplicative factors. Similarly, the loop induced decay $h \rightarrow \gamma \gamma$ will now draw an additional contribution from the charged scalars. Some recent investigations in this area can be found in [62-74]. Also, model-independent analysis of the data, which impose constraints on non-SM couplings of the scalar discovered, have to allow such contributions [75-80]. In order to check the consistency of a $2 \mathrm{HDM}$ with the measured rates in various channels, we theoretically compute the signal strength $\mu^{i}$ for the $i$-th channel using the relation:

$$
\mu^{i}=\frac{R_{\text {prod }} \times R_{\text {decay }}^{i}}{R_{\text {width }}} .
$$

Here $R_{\text {prod }}, R_{\text {decay }}^{i}$ and $R_{\text {width }}$ denote respectively the ratios of the theoretically calculated production cross section, the decay rate to the $i$-th channel and the total decay width for a $\sim 125 \mathrm{GeV}$ Higgs to their corresponding SM counterparts. Thus, our analysis strategy is to generate a region in parameter space allowed by the constraints coming from vacuum stability, perturbative unitarity and electroweak precision data. We subsequently compute $\mu^{i}$ for each point in that allowed region and compare them to the experimentally measured signal strengths, $\hat{\mu}^{i}$, supplied by the LHC. This exercise carves out a sub-region, which is allowed by the recent Higgs data, from the previously obtained parameter space. We have implemented the Runge-Kutta algorithm to solve the RG equations through our own code. The oblique parameters and the signal strengths to various channels have been computed using standard formulae available in the literature. Moreover, the consistency of the obtained results have been checked using the public code $2 \mathrm{HDMC}$ [81] at various parameter points.

For our numerical analysis, we have taken gluon fusion to be the dominant production mode for the SM-like Higgs. ${ }^{3}$ As for the subsequent decays of $h$, we have considered all the decay channels mentioned in table 1 . We use $1 \sigma$ allowed ranges of $\hat{\mu}^{i}$.

\footnotetext{
${ }^{3}$ While other channels such as vector boson fusion (VBF) and associated Higgs production with $\mathrm{W} / \mathrm{Z}$ (VH) have yielded data in the $8 \mathrm{TeV}$ run, the best fit signal strengths are still dominated by the gluon fusion channel. Here our primary task is to check the high scale validity of the 2HDM. In that approximation, the $\mathrm{K}$-factors in $\sigma$ and $\sigma_{\mathrm{SM}}$ are taken to be the same.
} 


\begin{tabular}{|c|c|c|c|}
\hline Channel & Experiment & $\hat{\mu}$ & Energy in TeV $\left(\right.$ Luminosity in $\left.\mathrm{fb}^{-1}\right)$ \\
\hline \multirow{2}{*}{$h \rightarrow \gamma \gamma$} & ATLAS & $1.55_{-0.28}^{+0.33}$ & $7(4.8)+8(20.7)$ \\
\cline { 2 - 4 } & CMS & $1.13_{-0.24}^{+0.24}$ & $7(5.1)+8(19.6)$ \\
\hline \multirow{2}{*}{$h \stackrel{Z Z^{*}}{\rightarrow} 4 l$} & ATLAS & $1.43_{-0.35}^{+0.40}$ & $7(4.6)+8(20.7)$ \\
\cline { 2 - 4 } & CMS & $1.00_{-0.29}^{+0.29}$ & $7(5.1)+8(19.7)$ \\
\hline \multirow{2}{*}{$h \stackrel{W W^{*}}{\longrightarrow} 2 l 2 \nu$} & ATLAS & $0.99_{-0.28}^{+0.31}$ & $7(4.6)+8(20.7)$ \\
\cline { 2 - 4 } & CMS & $0.83_{-0.21}^{+0.21}$ & $7(4.9)+8(19.4)$ \\
\hline \multirow{2}{*}{$h \rightarrow b \bar{b}$} & ATLAS & $0.20_{-0.60}^{+0.70}$ & $7(4.7)+8(20.3)$ \\
\cline { 2 - 4 } & CMS & $0.91_{-0.49}^{+0.49}$ & $7(5.1)+8(18.9)$ \\
\hline \multirow{2}{*}{$h \rightarrow \tau \bar{\tau}$} & ATLAS & $1.4_{-0.40}^{+0.50}$ & $8(20.3)$ \\
\cline { 2 - 4 } & CMS & $0.91_{-0.27}^{+0.27}$ & $7(4.9)+8(19.7)$ \\
\hline
\end{tabular}

Table 1. The signal strengths in various channels with their $1 \sigma$ uncertainties.

\section{Results with exact discrete symmetry}

In this section, we set out to obtain the allowed parameter space of a Type-II 2HDM having an exact $\mathbb{Z}_{2}$ symmetry consistent with the various theoretical and collider constraints described above. In this particular case, one naturally has $m_{12}=0, \lambda_{6}, \lambda_{7}=0$. Thus, we scan over the quartic couplings $\lambda_{i}(i=1, \ldots, 5)$ within their perturbative limits $\left(\lambda_{1,2} \in\right.$ $[0,4 \pi]$ and $\left.\lambda_{3,4,5} \in[-4 \pi, 4 \pi]\right)$ and allow them to evolve from a low scale to a higher scale, designated by $\Lambda_{\mathrm{UV}}$. The RG equations for the evolution of all the $2 \mathrm{HDM}$ couplings are listed in appendix A. In our analysis, the scale from which the evolution starts, has been chosen to be the top quark pole mass $M_{t}=173.1 \mathrm{GeV}$. This pins down the values of the Yukawa couplings at that scale through the relations $y_{t}\left(M_{t}\right)=\sqrt{2} m_{t}\left(M_{t}\right) / v_{2}$ and $y_{i}\left(M_{t}\right)=\sqrt{2} m_{i}\left(M_{t}\right) / v_{1}$ for $i=b$ and $\tau$. Here $m_{j}\left(M_{t}\right)$ refers to the running mass of the $j$-th fermion at the scale $M_{t}$ in $\overline{M S}$ scheme. We choose $m_{t}\left(M_{t}\right), m_{b}\left(M_{t}\right)$ and $m_{\tau}\left(M_{t}\right)$ to be $163.30,4.20$ and $1.77 \mathrm{GeV}$ respectively $[82,83]$.

We obtain the allowed values of $\lambda_{i}\left(M_{t}\right)(i=1, \ldots, 5)$ which, in course of evolution towards $\Lambda_{\mathrm{UV}}$, satisfy all the constraints of perturbativity, unitarity and vacuum stability at all intermediate scales. We choose $\Lambda_{\mathrm{UV}}=1 \mathrm{TeV}$ and $\tan \beta=2$ as an appropriate benchmark.

We display our scan results as allowed regions of parameter space in $m_{H}-m_{A}$ plane as well in the $m_{H^{ \pm}}-\alpha$ plane as shown in figure 1 . The oblique parameters play a role in restricting the splitting between the masses. Moreover, demanding perturbative unitarity and vacuum stability up to the $\mathrm{TeV}$ scale causes the allowed region to shrink further. In other words, for any value of the masses not within the allowed region, the quartic couplings are such that, if they are used as initial conditions in the RG equations, they would violate perturbative unitarity or vacuum stability below the TeV scale. For example, vacuum stability up to the $\mathrm{TeV}$ scale puts an upper bound on $\left|\lambda_{5}\right|$ which in turn translates into an upper bound on $m_{A}$ (see eqs. (2.5a) and (3.1d)). The mixing angle $\alpha$ gets further constrained by the recent Higgs data. Since, values of $m_{H}$ and $m_{A}$ chosen do not play a 

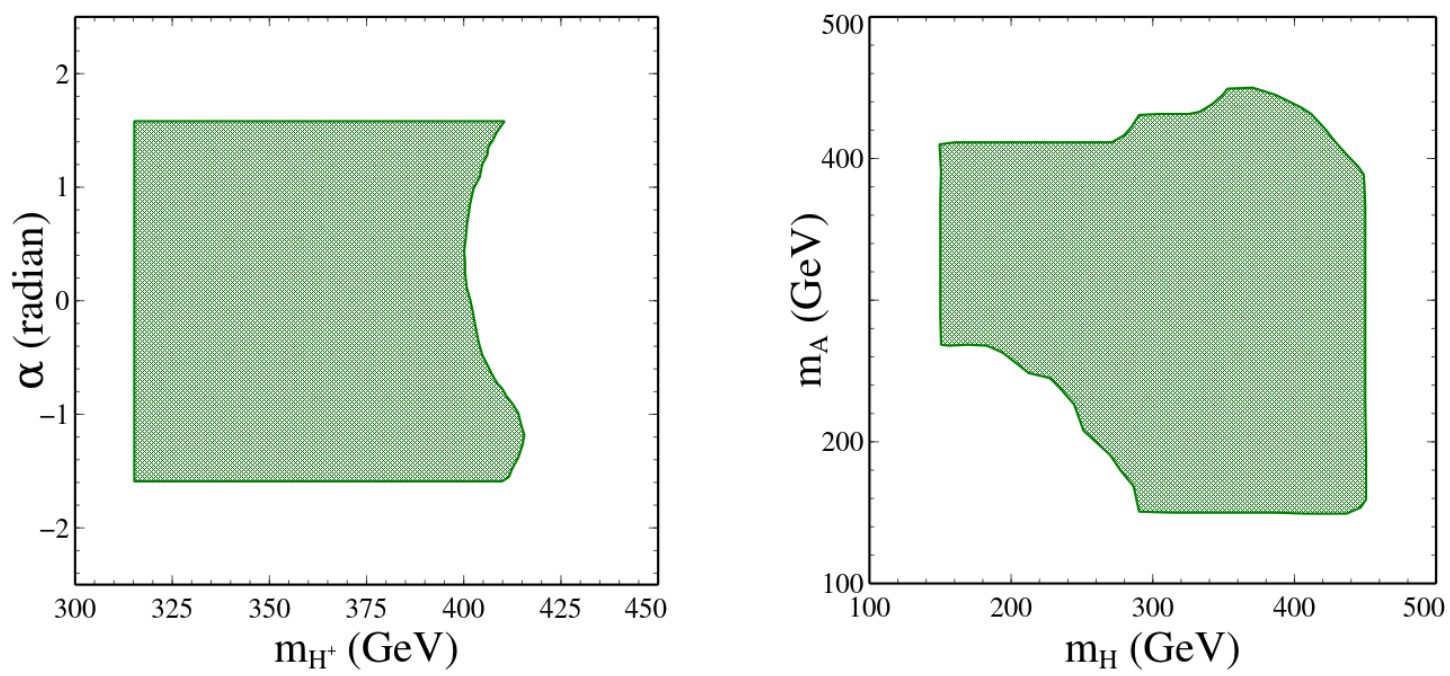

Figure 1. Theoretically allowed parameter spaces at $\Lambda_{\mathrm{UV}}=1 \mathrm{TeV}, \tan \beta=2$ and $m_{12}=0 \mathrm{GeV}$ for $M_{t}=173.1 \mathrm{GeV}$. The region in the figure on the left is allowed concomitantly with that in the figure on the right.
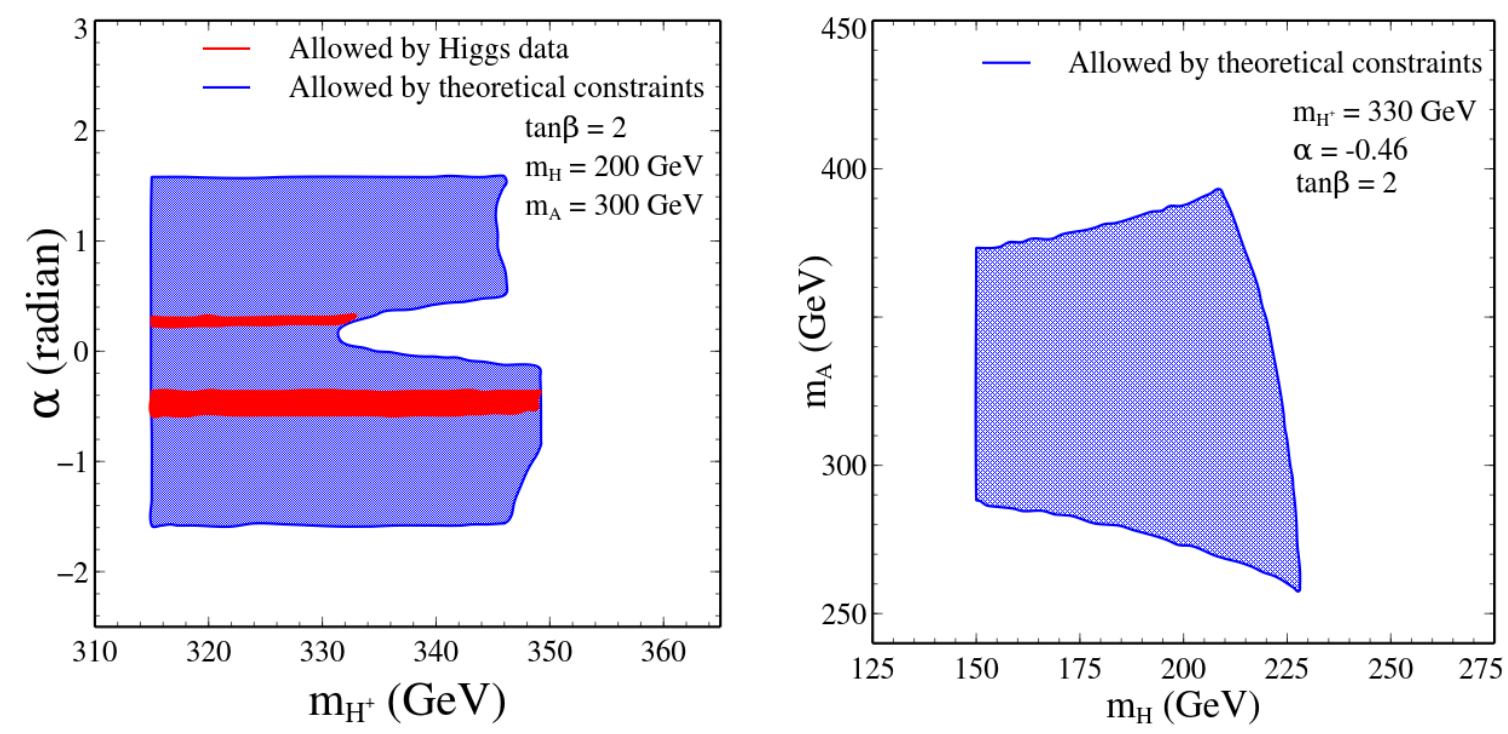

Figure 2. Region allowed in the $m_{H^{ \pm}-\alpha}$ and $m_{H^{-}} m_{A}$ planes, by the theoretical constraints and the recent Higgs data. In each case, the chosen benchmark values of the two other parameters are given in the legend.

role in modifying the Higgs signal strengths, we choose a benchmark $m_{H}=200 \mathrm{GeV}$ and $m_{A}=300 \mathrm{GeV}$ and project the allowed region in the $m_{H^{ \pm}}-\alpha$ plane, shown in figure 2 .

Recent data indicate that $M_{t}$ the top quark pole mass is [173.07 $\left.\pm 0.52 \pm 0.72\right] \mathrm{GeV}$ [52]. ${ }^{4}$ Different values for $M_{t}$ (within the allowed band) necessarily alter the running masses as well. However, choosing different values of the top quark mass does not cause any noticeable

\footnotetext{
${ }^{4}$ We have used the allowed range of the top quark pole mass as given in the above reference. The allowed range changes slightly, according to the most recent result [84].
} 
change to the allowed region in the parameter space of scalar masses and mixing angle. Since in this case, the RG running of the quartic couplings takes place over a relatively shorter length of energy scale, i.e., from the electroweak scale to $10 \mathrm{TeV}$, the evolution trajectories corresponding to different values of the top mass do not diverge apart from each other. For example, it has been checked that the allowed space in terms of masses, where we have used $M_{t}=173.1 \mathrm{GeV}$, remains almost identical if $M_{t}$ takes any value between 171.0 and $175.2 \mathrm{GeV}$.

We thus can say that, in case of exact $\mathbb{Z}_{2}$ symmetry, the uncertainty in the top quark mass measurement has almost no bearing on the allowed region of the parameter space. This result alerts us to a more important one that we obtain in the next sections, namely, the high scale validity of the $2 \mathrm{HDM}$ irrespective of the measured value of the top quark mass.

For $\beta-\alpha=\pi / 2$, the $2 \mathrm{HDM}$ couplings of the $125 \mathrm{GeV}$ Higgs to fermions and gauge bosons are the same as the SM ones. In that case, the Higgs signal strengths to various channels should match with the corresponding SM ones. Figure 2 shows an allowed band around $\alpha=\beta-\pi / 2=-0.46$ thus validating this observation. Over the entire region marked with red in figure $2, \cos (\beta-\alpha)$ is very small. As a result, $m_{H}=200 \mathrm{GeV}$ is not ruled out by the LHC data, since the $Z Z$ and $W W$ decay modes of $H$ are suppressed.

To illustrate the RG running of the various couplings, the vacuum stability conditions and the LQT eigenvalues, we choose the following initial conditions,

$$
\lambda_{1}\left(M_{t}\right)=1.33, \lambda_{2}\left(M_{t}\right)=0.90, \lambda_{3}\left(M_{t}\right)=4.08, \lambda_{4}\left(M_{t}\right)=-2.13 \text {, and } \lambda_{5}\left(M_{t}\right)=-1.79 \text {. }
$$

This choice of boundary conditions for our illustration is aimed at keeping $\lambda_{1}$ as low as possible, with $m_{h}$ in the right range. We want to show that even with such a choice, the theory violates perturbativity and unitarity below $10 \mathrm{TeV}$. Thus the impossibility of this $2 \mathrm{HDM}$ with $m_{12}=0$ at high scale gets established. Figure 3 describes the RG running of $\lambda_{i}$ with the aforementioned low values as boundary conditions. These values correspond to,

$$
\begin{aligned}
m_{h} & =125.44 \mathrm{GeV}, \quad m_{H}=210.00 \mathrm{GeV}, \quad m_{H^{ \pm}}=345.00 \mathrm{GeV}, \\
m_{A} & =330.00 \mathrm{GeV}, \quad \alpha=0.95 \text { radian },
\end{aligned}
$$

which is an allowed point in the parameter space, as shown in figure 2 . Since $\lambda_{1}$ starts evolving from rather large a value, it rises steeply with the energy scale. For the value of $\tan \beta$ taken here, $y_{b}$ and $y_{\tau}$ have small initial values, and hence, they do not slow the evolution curve down to any appreciable extent (see eq. (A.2a)). On the other hand, $y_{t}$, being the dominant Yukawa coupling in this case, prevents $\lambda_{2}$ to rise as sharply as $\lambda_{1}$. The LQT eigenvalues (see eq. (B.3)) $a_{+}, b_{+}$and $c_{+}$evolve in a manner as shown in figure $3 \mathrm{~b}$. Also, the stability conditions remain positive during the course of evolution, as shown in figure 3c. A different initial condition which has a higher value of $\lambda_{1}\left(M_{t}\right)$ for instance, would lead to steeper evolution trajectories for the couplings. Hence, the overall conclusions regarding high-scale validity of this scenario would not alter.

This leads to the observation that the various $\lambda_{i}$ become non-perturbative below a scale of $10 \mathrm{TeV}$. Also, it is seen that the LQT eigenvalue $a_{+}$hits the unitarity limit faster 


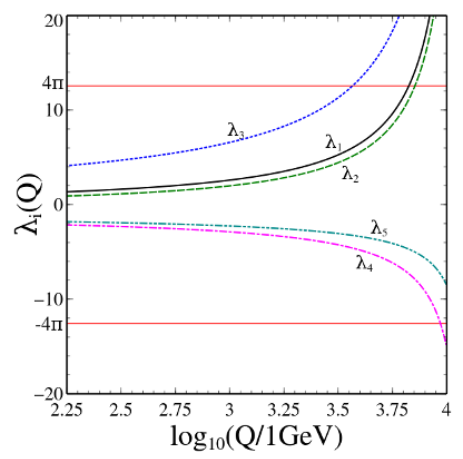

(a)

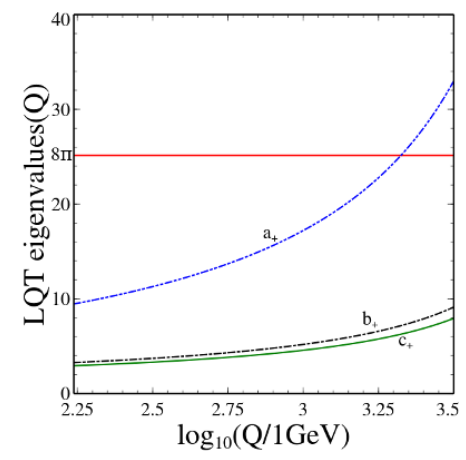

(b)

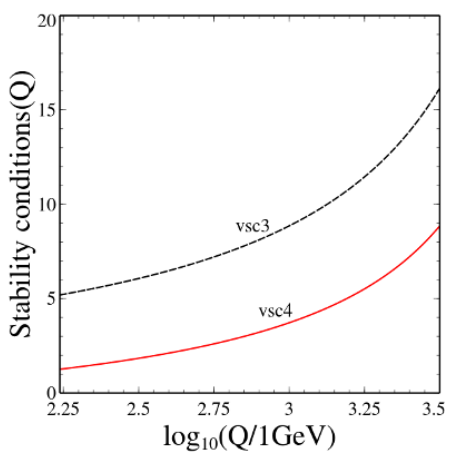

(c)

Figure 3. RG running of $\lambda_{i}$, the LQT eigenvalues and the stability conditions with the energy scale for $\tan \beta=2$ and $m_{12}=0$. The horizontal lines in the leftmost figure 3a denote the perturbative limit and unitarity limit in the second figure $3 \mathrm{~b}$. Also $a_{+}, b_{+}$and $c_{+}$in the second figure $3 \mathrm{~b}$ are the LQT eigenvalues explained in the appendix B. In the rightmost figure $3 \mathrm{c}, \mathrm{vsc} 3$ and vsc 4 represent the two stability conditions that are defined in eq. (3.1).

than the quartic couplings hit their perturbative limits. Thus, this example illustrates the interplay among perturbativity and unitarity in determining the UV fate of this scenario and it appears that unitarity often proves stronger as a constraint than perturbativity. It should also be noted that all plots in figures 1 and 2 use $\tan \beta=2$. This is because the quartic couplings cannot be kept in their perturbative limits for $\tan \beta \geq 3$. A wider scan over the parameter space corresponds to this observation. Also, one can generally conclude that in order to push the UV limit of $2 \mathrm{HDM}$ to higher scales, one must look beyond the exact $\mathbb{Z}_{2}$ symmetric case.

\section{$5 \quad$ Results with softly broken discrete symmetry}

This section illustrates the effects of the various constraints imposed on the model with non-zero $m_{12}$, i.e., in presence of a soft $\mathbb{Z}_{2}$ symmetry violating term. The $\mathrm{RG}$ runnings of the various couplings in the model are just like the ones in exact $\mathbb{Z}_{2}$ symmetric case, the only differences being in the expressions for the scalar masses as evident from eq. (2.5). We scan the model parameter space and look for points which satisfy all the constraints listed in section 3 up to $\Lambda_{\mathrm{UV}}=10^{3}, 10^{11}, 10^{16}, 10^{19} \mathrm{GeV}$ in figures 4 , and 6 . Validity of the model up to the TeV scale, Grand Unification scale, Planck scale as well as $\sqrt{M_{\mathrm{Pl}} M_{\mathrm{TeV}}}$ is addressed in this manner. ${ }^{5}$

\footnotetext{
${ }^{5}$ Here $M_{\mathrm{Pl}}$ and $M_{\mathrm{TeV}}$ represents the Planck scale and TeV scale respectively. The benchmarks chosen are $\tan \beta=2,10,20$ and $m_{12}=200,1000 \mathrm{GeV}$, which represent the electroweak and TeV scales. This also keeps the $2 \mathrm{HDM}$ spectrum within the ultimate reach of the LHC. Having $\tan \beta$ higher than in the previous section is possible in this case, so long as $m_{12}$ is correspondingly large, thus generating an acceptable $m_{h}$. For $\Lambda_{\mathrm{UV}}=10^{11}, 10^{16}, 10^{19} \mathrm{GeV}$, we project our allowed results as two dimensional contour plots in the $m_{H}-m_{A}$ and $m_{H^{ \pm}}-\alpha$ planes. In each row, the plots in the left- and right-hand sides represent concomitantly allowed regions. This choice pins down the $2 \mathrm{HDM}$ parameter space in terms of the physically measurable observables.
} 

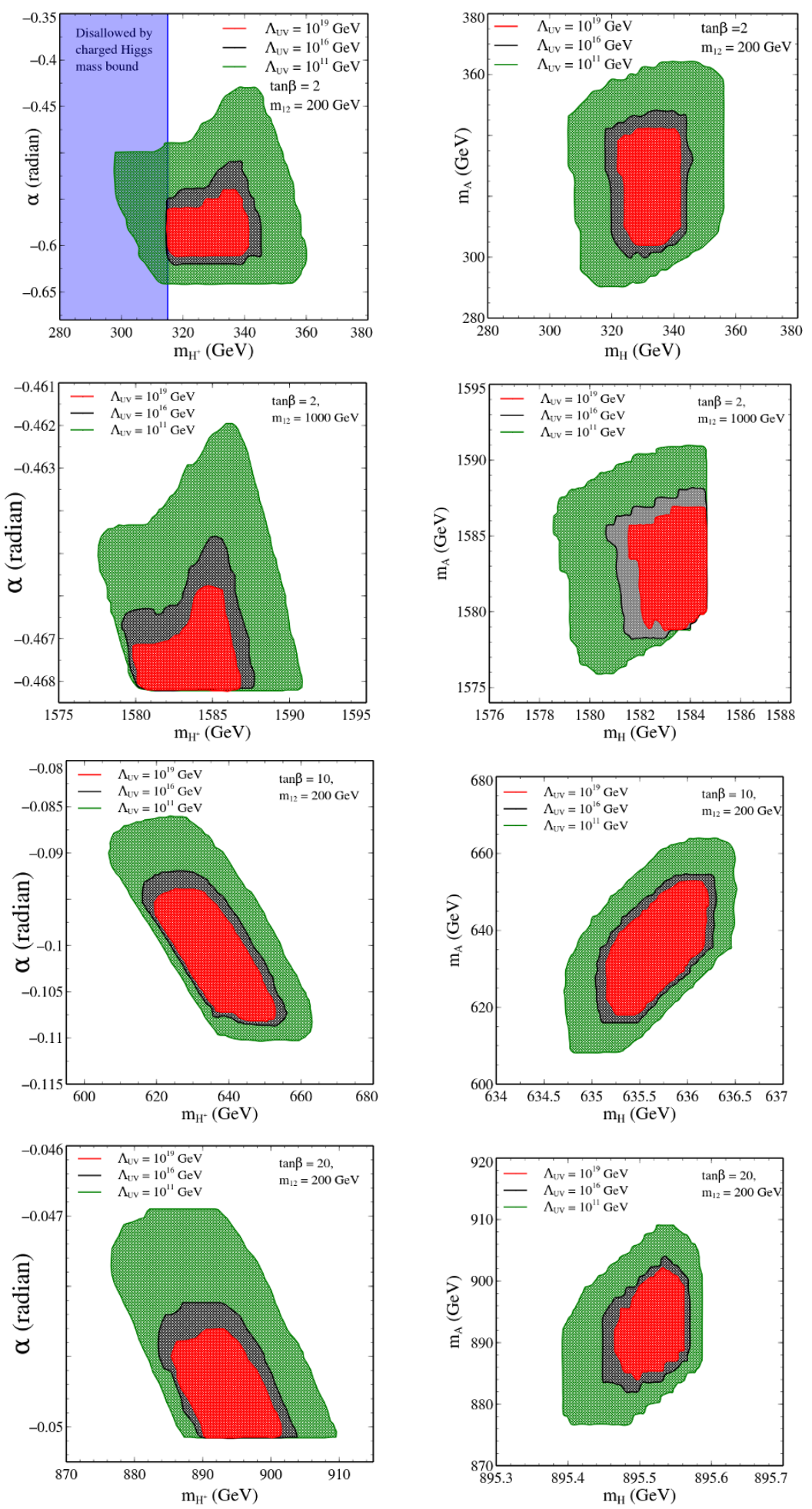

Figure 4. The allowed parameter spaces in the soft $\mathbb{Z}_{2}$ breaking case for $\Lambda_{\mathrm{UV}}=10^{11}$ (green), $10^{16}$ (grey) and $10^{19} \mathrm{GeV}$ (red). The $\tan \beta$ and $m_{12}$ values are shown in the plots. The shaded region (blue) in the top left figure denotes the exclusion coming from flavour constraints. 

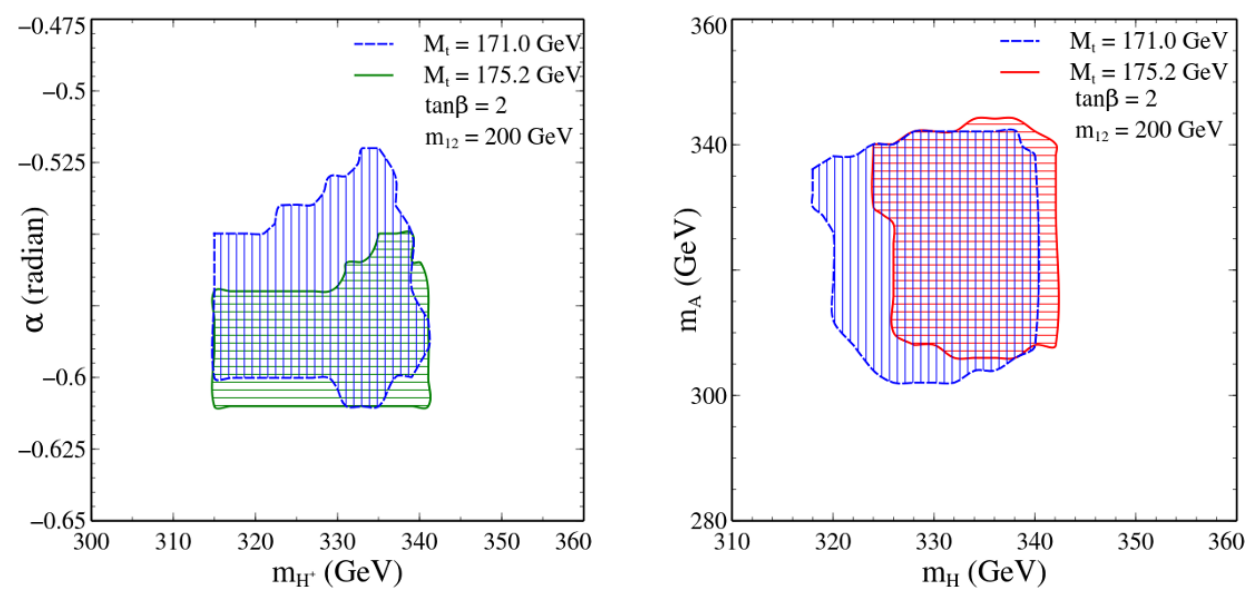

Figure 5. A comparison of the allowed parameter spaces at $\Lambda_{\mathrm{UV}}=10^{19} \mathrm{GeV}, \tan \beta=2$ and $m_{12}=1000 \mathrm{GeV}$ for two values of $M_{t}$, in the soft $\mathbb{Z}_{2}$ breaking case.

The results in the figure show that as we go higher in $\Lambda_{\mathrm{UV}}$, the allowed parameter space shrinks. The splitting amongst the scalar masses becomes the narrowest at the Planck scale, albeit being dependent on the values of $\tan \beta$ and $m_{12}$.

An inspection of the results so obtained shows that as $\Lambda_{\mathrm{UV}}$ is pushed towards higher scales, the allowed parameter space shrinks, and finally at the Planck scale, it is most constrained. For example, for $\tan \beta=10, m_{12}=200 \mathrm{GeV}$ and $\Lambda_{\mathrm{UV}}=10^{19} \mathrm{GeV}$, the masses (in $\mathrm{GeV}$ ) are observed to lie in the following range,

$$
m_{H} \in[635,636], m_{H^{ \pm}} \in[619,652], m_{A} \in[618,653] .
$$

We note here that though $m_{12}$ does not appear in the RG equations themselves, it indirectly puts constraints on $\lambda_{i}$ through the mass constraints.

Note that since $\tan \beta$ determines the initial conditions for the Yukawa couplings, it does affect the RG running of $\lambda_{i}$. Although $m_{b}\left(M_{t}\right)$ and $m_{\tau}\left(M_{t}\right)$ are small compared to $m_{t}\left(M_{t}\right)$, for a high $\tan \beta, y_{b}\left(M_{t}\right)$ could be comparable to $y_{t}\left(M_{t}\right)$. This is the main motivation behind our choosing $\tan \beta=20$. A change in the top quark mass is expected to modify the obtained parameter space to a considerable extent. This fact is illustrated in figure 5 where we choose $M_{t}=171.0,175.2 \mathrm{GeV}$ and highlight the difference in the parameter spaces so obtained. In the subsequent sections, we keep $M_{t}=173.1 \mathrm{GeV}$.It may be argued that in determining the high scale validity of the model, the relatively less crucial role played here by the top quark mass is just due to the larger number of free parameters in the $2 \mathrm{HDM}$ scenario. While this is true in a sense, the analysis reported in figure 5 was still required for the following reason. To counter the downward evolution of $\lambda_{2}$ due to the top quark Yukawa coupling (see eq. (A.2b)), the participation of the other $\lambda_{i}$ plays a role. However, large values of these parameters may again violate perturbative unitarity, and in turn prevent one from extending the theory to high energy scales. The lesson to learn from figure 5 is that valid regions in the parameter space can be found, which survive the above tug-of-war. Consequently, a Type-II 2HDM may hold true till the Planck scale without any additional new physics, even for high-end values of the top quark mass. 

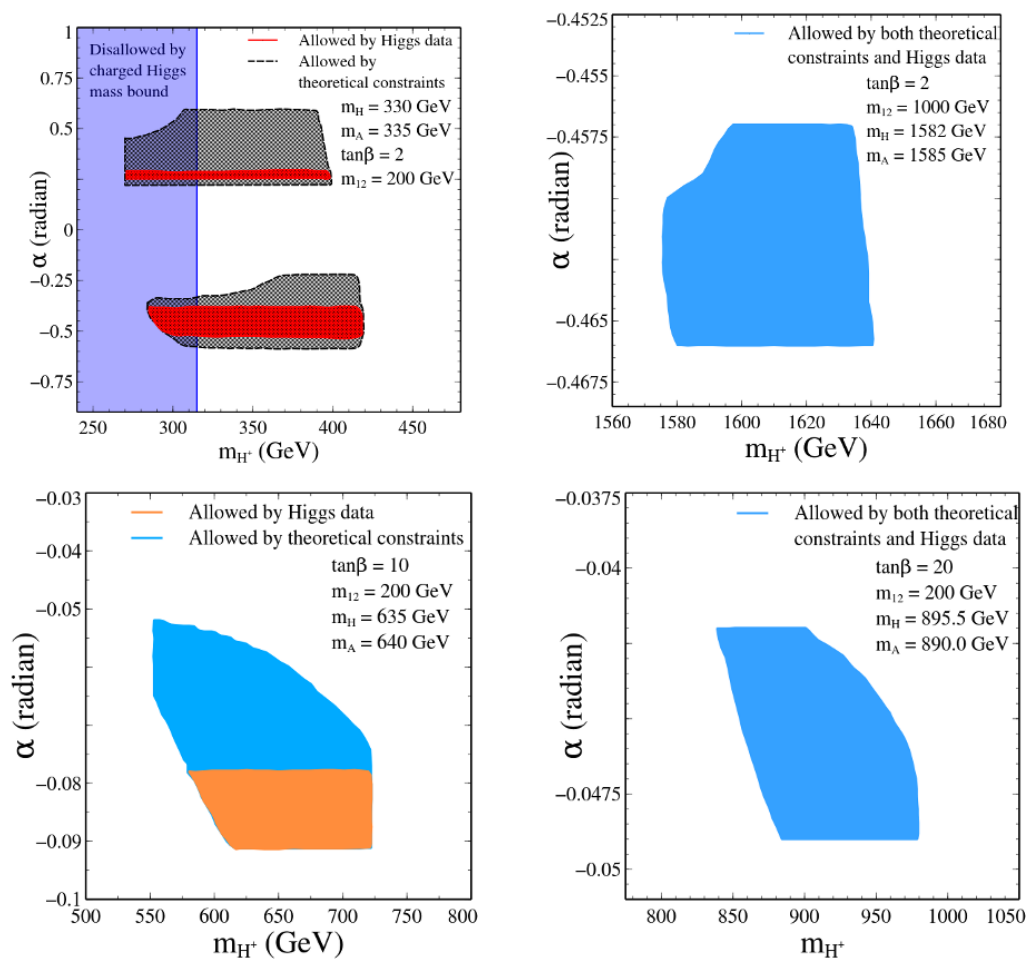

Figure 6. Regions in the $m_{H^{ \pm}}-\alpha$ plane allowed by the Higgs data in the soft $\mathbb{Z}_{2}$ breaking case.

The impact of the recent LHC data on the parameter space already allowed by the theoretical constraints is shown in figure 6 . In this case, we pick up benchmark values of $m_{H}$ and $m_{A}$ suitably to avoid the direct search constraints. In addition, these benchmarks are chosen from a region satisfying the theoretical constraints up to the Planck scale. The 2HDM decay widths are sensitive to the mixing angles and the charged scalar mass and the collider constraints carve out a subregion in the $m_{H^{ \pm}}-\alpha$ plane.

The figure shows allowed bands around $\alpha=\beta-\pi / 2$ in each case. Note that for scalar masses $\sim 1 \mathrm{TeV}$ or more, the entire region allowed by the theoretical constraints is favoured by the Higgs data. This is precisely due to the decoupling of the heavier degrees of freedom from the theory.

A small enough initial value of $\lambda_{2}$ causes $\lambda_{2}(Q)$ to turn negative at some scale affecting the vacuum stability of the theory thereby. To illustrate the point better, we choose an initial condition,

$$
\lambda_{1}\left(M_{t}\right)=0.03, \lambda_{2}\left(M_{t}\right)=0.39, \lambda_{3}\left(M_{t}\right)=0.49, \lambda_{4}\left(M_{t}\right)=-0.50 \text { and } \lambda_{5}\left(M_{t}\right)=0.03
$$

for the quartic couplings at $\tan \beta=2$ and $m_{12}=1000 \mathrm{GeV}$, out of the allowed set of couplings which obey all the imposed constraints up to the $\Lambda_{\mathrm{UV}}=10^{19} \mathrm{GeV}$. These quartic couplings expressed in terms of the masses and the mixing angle become,

$$
\begin{aligned}
m_{h} & =124.78 \mathrm{GeV}, \quad m_{H}=1582.31 \mathrm{GeV}, \quad m_{H^{ \pm}}=1585.64 \mathrm{GeV}, \\
m_{A} & =1580.56 \mathrm{GeV}, \quad \alpha=-0.466 \mathrm{radian} .
\end{aligned}
$$



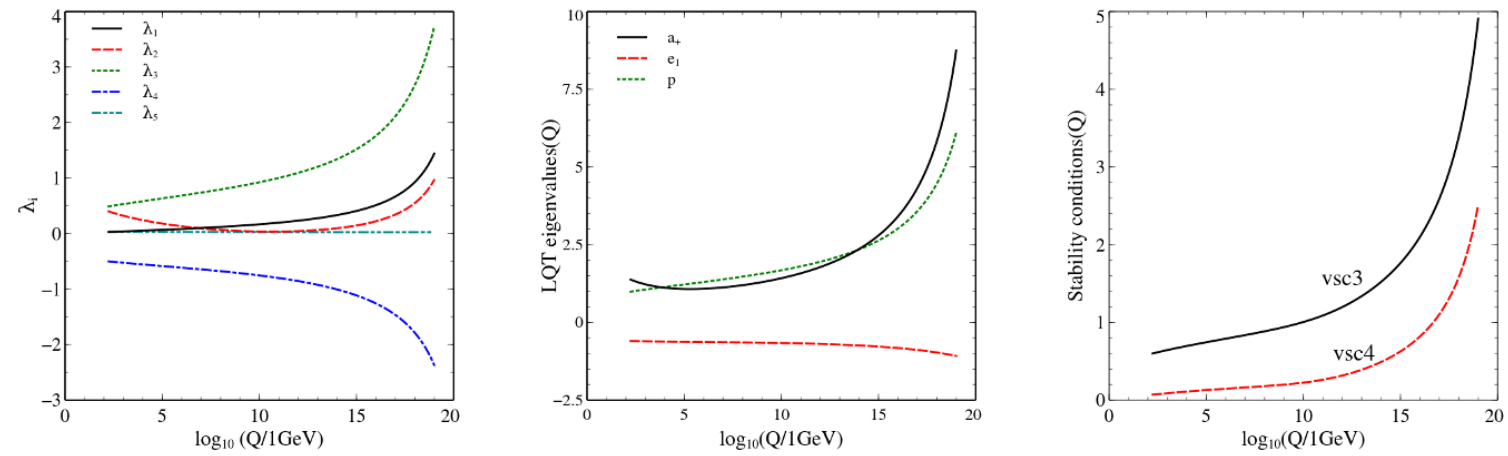

Figure 7. RG running of $\lambda_{i}$, the LQT eigenvalues and the stability conditions with the energy scale for $\tan \beta=2$ and $m_{12}=1000 \mathrm{GeV}$ in the soft $\mathbb{Z}_{2}$ breaking case.

We display the RG running of the $\lambda_{i}$, the stability conditions and the LQT eigenvalues in figure 7. This choice of sample boundary conditions here is guided by a consideration complimentary to that of figure 3 . Here we show that it is possible to identify points in the parameter space, which correspond to the quartic couplings avoiding any perturbativity, unitarity or vacuum stability constraints all the way up to the Planck scale. As indicated in figure $7, a_{+}(Q)$ grows most sharply amongst the other LQT eigenvalues and hence violates unitarity just after crossing the Planck scale in this case. Thus it turns out that $\left|a_{+}(Q)\right| \leq 8 \pi$ proves to be the strongest constraint in determining an upper bound on $\left|\lambda_{i}\right|$.

The most important observation that emerges from this part of the study is that the 2HDM can be valid all the way up to the GUT scale or even the Planck scale without the intervention of any new physics. This is true even if the top quark mass is at the upper end of the currently allowed range. The additional quartic couplings can counterbalance the effect of the Yukawa coupling threatening vacuum stability, while still remaining acceptable from the standpoint of perturbativity. It is seen that we get allowed parameter space for $\Lambda_{\mathrm{UV}}=10^{19} \mathrm{GeV}$ corresponding to several values of $\tan \beta$ and $m_{12}$. There is, however, a noticeable correlation - large $m_{12}$ tends to favour small values of $\tan \beta$. For too large an $m_{12}$, the contribution of the extra scalars decouples from the theory. In that case, the RG running of the couplings below that $m_{12}$ is governed by the SM beta functions. In that case, the stability of the electroweak vacuum is again more sensitive to the value chosen for $M_{t}$. This has been explicitly checked, for example with $m_{12}=10^{5} \mathrm{GeV}$.

The strong coupling constant affects our analysis by determining the initial condition for $g_{3}$. Current measurements yield a value $0.1184 \pm 0.0007$ for $\alpha_{s}\left(M_{Z}\right)$. In our analysis, we have used $\alpha_{s}\left(M_{Z}\right)=0.1184$ throughout. However, we demonstrate the effect of a $3 \sigma$ variation of $\alpha_{s}\left(M_{Z}\right)$ on the running of $\lambda_{2}$, the quartic coupling where the effect is expected to be more pronounced compared to the other ones.

We took $\lambda_{2}\left(M_{t}\right)=0.39$ in figure 8 . It is seen that the RG running is not significantly altered even by a $3 \sigma$ variation of $\alpha_{s}\left(M_{Z}\right)$. Hence, for any value of $\alpha_{s}\left(M_{Z}\right)$ within this band, the parameter spaces will not change in a major fashion, and whatever constraints apply to $\lambda_{2}\left(M_{t}\right)$ will continue to be valid rather insensitively to $\alpha_{s}\left(M_{Z}\right)$. 


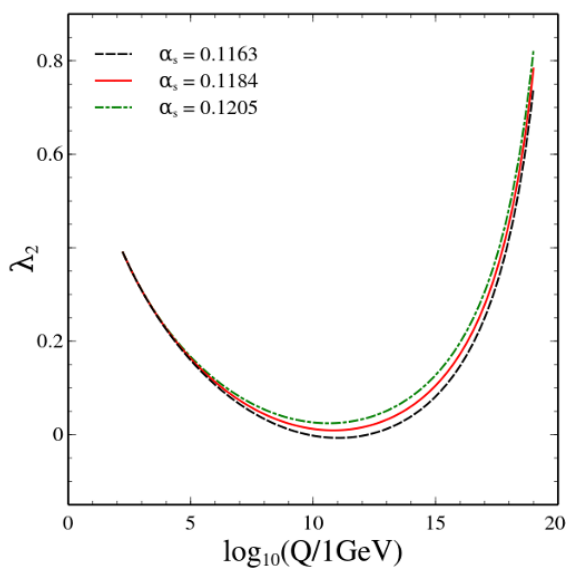

Figure 8. Running of $\lambda_{2}$ for three different values for $\alpha_{s}\left(M_{Z}\right)$ in the soft $\mathbb{Z}_{2}$ breaking case.

The implication of having a complex $m_{12}$ in the scalar potential $[38,39]$ is also investigated here. We rewrite the quadratic part of the scalar potential as,

$$
V_{\text {quad }}\left(\Phi_{1}, \Phi_{2}\right)=m_{11}^{2} \Phi_{1}^{\dagger} \Phi_{1}+m_{22}^{2} \Phi_{2}^{\dagger} \Phi_{2}-\left|m_{12}^{2}\right|\left(e^{i \delta} \Phi_{1}^{\dagger} \Phi_{2}+e^{-i \delta} \Phi_{2}^{\dagger} \Phi_{1}\right) .
$$

The quartic couplings are kept real as in the previous case. The presence of an arbitrary phase $\delta$ in $m_{12}^{2}$, leads to a charged scalar $H^{+}$, three neutral scalars $H_{1}, H_{2}$ and $H_{3}$ which are not eigenstates of $\mathrm{CP}$, and of course the charged and neutral Goldstone bosons. The masses of the neutral scalars can not be obtained in closed form in this case, rather, the corresponding mass matrix has to be diagonalised numerically. In the process of doing that, we choose the lightest neutral scalar, say $H_{3}$ to be around $125 \mathrm{GeV}$ and the charged scalar to have a mass higher than $315 \mathrm{GeV}$. The quartic couplings satisfying these conditions are selected and are further constrained by the imposition of the theoretical constraints under RG.

We have chosen the values $\delta=\frac{\pi}{4},\left|m_{12}\right|=200 \mathrm{GeV}$ and $\tan \beta=2$ as benchmark. This choice is illustrative. Constraints on the phase from, say, the electron dipole moment requires full evaluation at each point in the parameter space. For more discussions, we refer the reader to [85]. Scatter plots in mass planes are presented in figure 9. For higher $\Lambda_{U V}$, the bounds on the scalar masses become tighter. To make the effect of the added phase in changing the scalar masses, we also show the mass bounds in the situation with $\delta=0$. We would like to emphasize that it is not our purpose here to scan the allowed range of $\delta$ for different values of the mass parameters and quartic couplings. The point that we make is that the validity of this $2 \mathrm{HDM}$ up to high scales holds even with a CP-violating phase in the potential. $\delta=\frac{\pi}{4}$ is chosen as a benchmark for this demonstration. A detailed study of the $\delta$ dependence of the allowed parameter space and its phenomenological implications is the subject of a separate project.

Our observation therefore is that the regions in the parameter space of a 2HDM, consistent with UV completion at the GUT/Planck scale, are dependent on the phase of the complex parameter(s) of the scalar potential. Together with the less crucial role played by the top mass uncertainty, this is the other important lesson to take home from this section. 

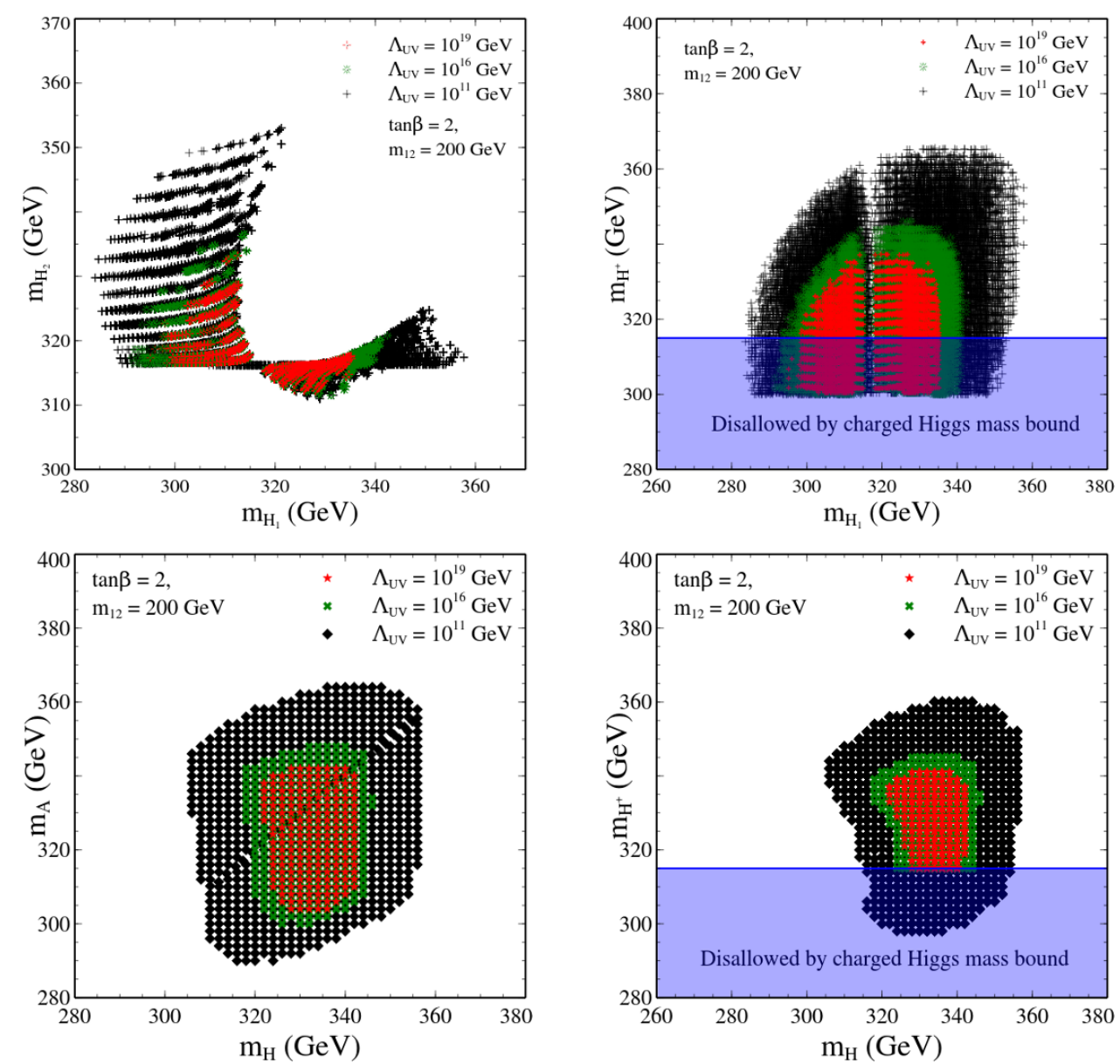

Figure 9. The allowed regions in mass plane as a function of $\Lambda_{\mathrm{UV}}$ in the soft $\mathbb{Z}_{2}$ breaking case. The upper and lower two plots correspond to $\delta=\frac{\pi}{4}$ and $\delta=0$ respectively.

\section{Results with quartic terms breaking the discrete symmetry}

We now come to the last part of our study where the $\mathbb{Z}_{2}$ symmetry is broken both at the soft and hard level (i.e., $m_{12}, \lambda_{6}, \lambda_{7} \neq 0$ ). In this case however, the RG running of the various couplings in the theory is different with respect to the soft breaking case, owing to the introduction of $\lambda_{6}$ and $\lambda_{7}$ (see appendix $\mathrm{A}$ for the complete set of RG equations). While scanning the $\lambda_{i}$ parameter space, we try to reduce the number of free parameters so that the analysis does not become unwieldy. We therefore fix some parameters studied earlier within their allowed ranges. In this spirit, we choose $\lambda_{1}\left(M_{t}\right)=0.02$ and $\lambda_{6}\left(M_{t}\right)=\lambda_{7}\left(M_{t}\right)$ for computational convenience. $\lambda_{1}\left(M_{t}\right)$ has been deliberately chosen to be small so that it respects perturbative unitarity even up to the Planck scale. As in the plots shown previously, we present the allowed regions in the $m_{H}-m_{A}$ and $m_{H^{ \pm}}-\alpha$ planes which satisfy all the conditions up to $\Lambda_{\mathrm{UV}}=10^{3}, 10^{11}, 10^{16}, 10^{19} \mathrm{GeV}$. The benchmarks are $\tan \beta=2,10,20$ and $m_{12}=200,1000 \mathrm{GeV}$. The results of the scans are shown in figure 10 .

The range over which the scalar masses are distributed can be seen in figure 10. We note that for $m_{12}=1 \mathrm{TeV}$, the resulting scalar spectrum is almost degenerate. This is 

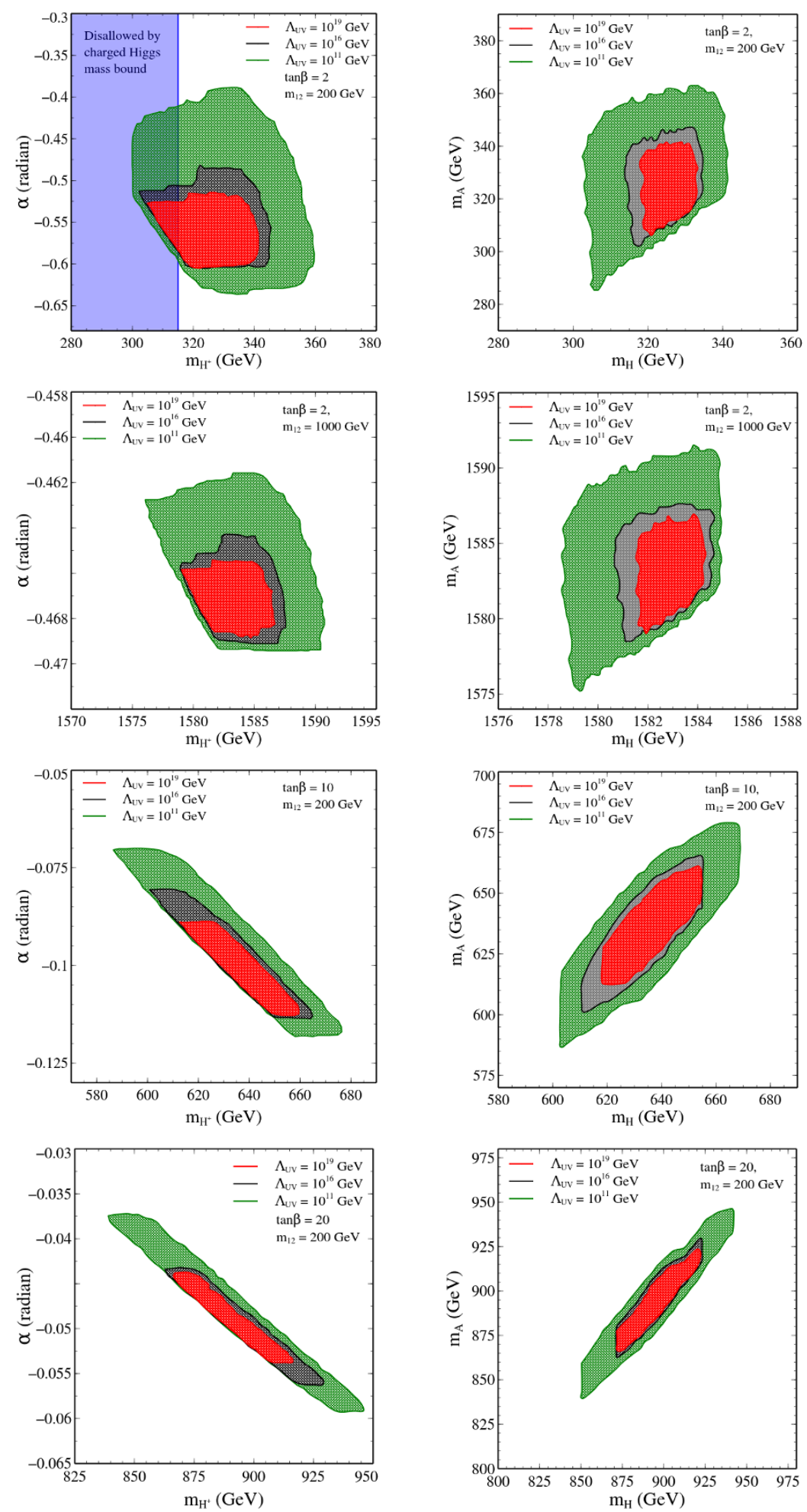

Figure 10. The allowed parameter spaces for $\Lambda_{\mathrm{UV}}=10^{11}$ (green), $10^{16}$ (grey) and $10^{19} \mathrm{GeV}$ (red), in the $\lambda_{6}, \lambda_{7} \neq 0$ case. The $\tan \beta$ and $m_{12}$ values are shown in the plots. 

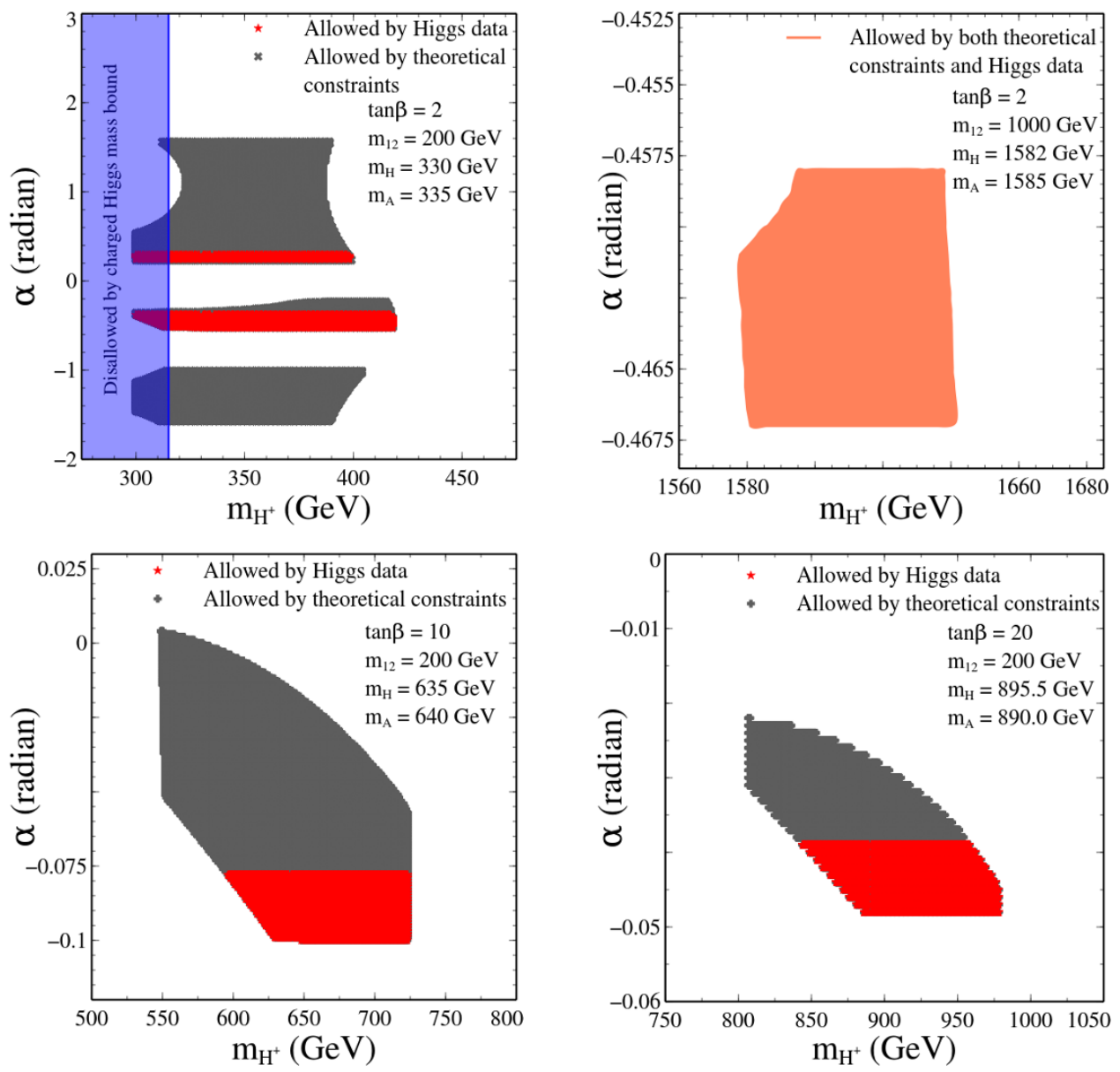

Figure 11. Results for $\Lambda_{\mathrm{UV}}=1 \mathrm{TeV}$, in the $\lambda_{6}, \lambda_{7} \neq 0$ case. The regions in red denote the part of the parameter space allowed by the Higgs data.

precisely due to the fact that the theoretical constraints pin down the allowed values of $\lambda_{i}$ to a rather constricted range which also constrains the scalar masses and also the mixing angle in turn. Similarly, for $m_{12}=200 \mathrm{GeV}$, the variation allowed in $\lambda_{i}$ causes a variation of $\sim 100 \mathrm{GeV}$ in the mass of a scalar.

In the case where $\Lambda_{\mathrm{UV}}=10^{3} \mathrm{GeV}$, we show the subregions in the parameter spaces which are also allowed by the recent Higgs data. Similar to the previous sections, the results have been given in terms of allowed regions in the $m_{H^{ \pm}}-\alpha$ plane for specific benchmark values of $m_{H}$ and $m_{A}$. The major constraint, however, comes from the signal strength corresponding to $h \rightarrow \gamma \gamma$. It is clearly seen in figure 11 that $m_{12}=1000 \mathrm{GeV}$ allows for a bigger region in the parameter space that is allowed by the Higgs data at $1 \sigma$ level, compared to what $m_{12}=200 \mathrm{GeV}$ does. This is obviously expected, given the fact that a high value of $m_{12}$ takes the theory towards the decoupling limit, and thus the $125 \mathrm{GeV}$ Higgs becomes SM-like. Hence, the bounds predicted on the scalar masses and the mixing angle together by the theoretical and collider constraints could be well tested in the next run of the LHC. 

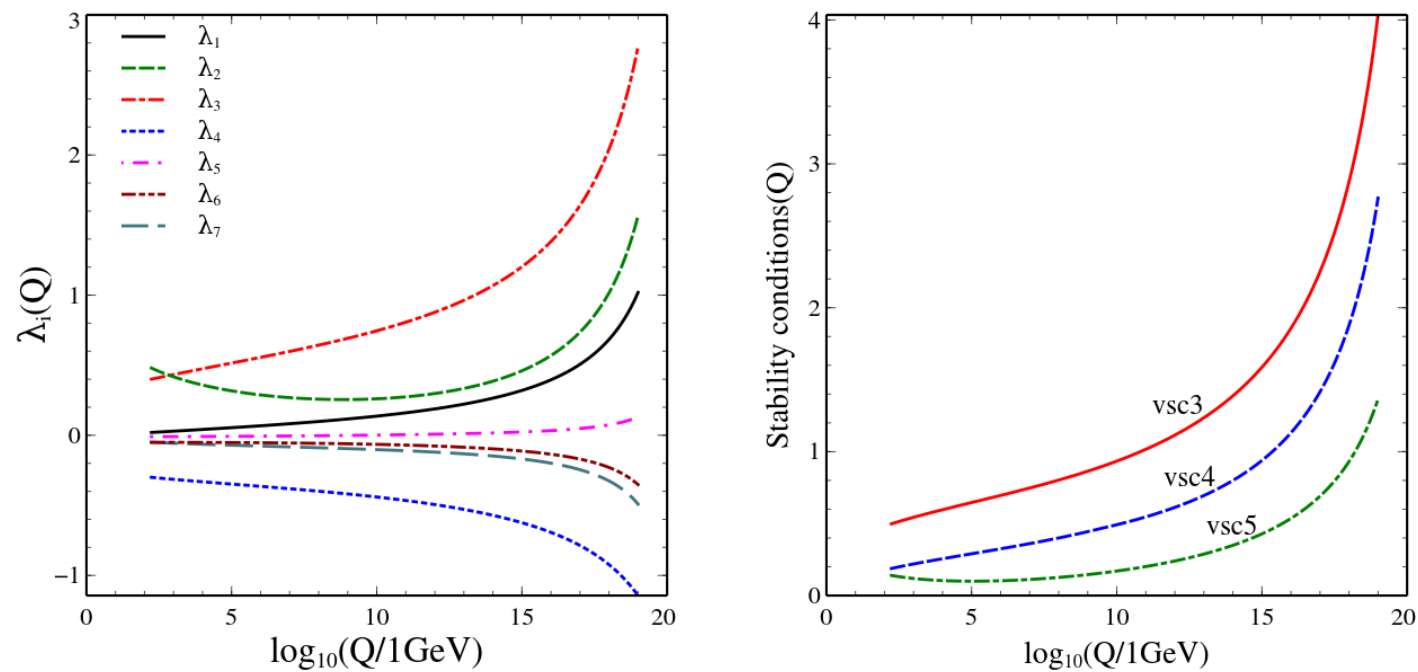

Figure 12. RG running of $\lambda_{i}$ and the stability conditions with the energy scale for $\tan \beta=2$ and $m_{12}=1000 \mathrm{GeV}$, in the $\lambda_{6}, \lambda_{7} \neq 0$ case.

We demonstrate the UV completion of the hard $\mathbb{Z}_{2}$ violating case by showing the RG evolution of the various quartic couplings and stability conditions up to $\Lambda_{\mathrm{UV}}=10^{19} \mathrm{GeV}$. We choose the following initial conditions for the quartic couplings at $\tan \beta=2$ and $m_{12}=1000 \mathrm{GeV}$,

$$
\begin{array}{llcc}
\lambda_{1}\left(M_{t}\right)=0.02, & \lambda_{2}\left(M_{t}\right)=0.48, & \lambda_{3}\left(M_{t}\right)=0.40, & \lambda_{4}\left(M_{t}\right)=-0.30 \\
\lambda_{5}\left(M_{t}\right)=-0.01, & \lambda_{6}\left(M_{t}\right)=-0.05 & \text { and } & \lambda_{7}\left(M_{t}\right)=-0.05 .
\end{array}
$$

These particular initial conditions correspond to,

$$
\begin{aligned}
m_{h} & =124.62 \mathrm{GeV}, \quad m_{H}=1583.33 \mathrm{GeV}, \quad m_{H^{ \pm}}=1585.30 \mathrm{GeV}, \\
m_{A} & =1582.52 \mathrm{GeV}, \quad \alpha=-0.467 \text { radian },
\end{aligned}
$$

and is an allowed point in the parameter space corresponding to the benchmark $m_{12}=$ $1000 \mathrm{GeV}, \tan \beta=2$ and $\Lambda_{\mathrm{UV}}=10^{19} \mathrm{GeV}$. As explained just after eq. (4.1), the lowenergy boundary values in are just illustrative. In all our quantitative scans (shown in figures $1,2,4,5,6,9,10$ and 11) to determine high-scale validity, a wide range of such boundary conditions are employed. Thus there is nothing fine-tuned about eqs. (4.1), (5.2) and (6.1). As shown in figure 12, $\lambda_{3}$ increases most sharply whereas $\lambda_{2}$ first plunges down due to the effect of the $\mathcal{O}\left(y_{t}^{4}\right)$ term in the RG equation (see eq. (A.2b)) and then starts increasing. Choosing same initial conditions for $\lambda_{6}$ and $\lambda_{7}$ causes their evolutions to become fairly similar. In this section, it should be noted that the allowed parameter spaces found are not expected to be exhaustive as we have not scanned over all $\lambda_{i}\left(M_{t}\right)$ independently, rather, have put $\lambda_{1}\left(M_{t}\right)=0.02$ and $\lambda_{6}\left(M_{t}\right)=\lambda_{7}\left(M_{t}\right)$ while doing so. However, given the similar structure of the 1-loop beta functions of $\lambda_{6}$ and $\lambda_{7}$ (see eq. (A.2f) and (A.2g)), the bounds obtained on them would have not substantially changed even if an independent scanning would have been done. 


\section{Summary and conclusions}

We set out to investigate the high-scale behaviour of a 2HDM. The results are illustrated in the context of a Type-II scenario. We have used the theoretical constraints of perturbativity, unitarity and vacuum stability to constrain the parameter space of the model. The relatively less stringent constraints from oblique parameters, and also the LHC constraints on the signal strength in each decay channel of a Higgs around $125 \mathrm{GeV}$ have also been taken into account.

We find that a $2 \mathrm{HDM}$ with a discrete $\mathbb{Z}_{2}$ symmetry (thereby forbidding some crossterms in the two doublets in the potential) cannot be valid beyond $10 \mathrm{TeV}$, since otherwise the requirement of keeping one neutral scalar mass around $125 \mathrm{GeV}$ cannot be met. With the discrete symmetry broken, on the other hand, it is possible to fulfill all the constraints over a much larger region of the parameter space. Thus the theory with a 2HDM can distinctly be valid up to energies as high as $10^{16} \mathrm{GeV}$ or even the Planck scale, without the intervention of any additional physics. This feature holds irrespectively of the uncertainty in the measured value of the top quark mass, which is in contrast to what is expected in the standard model with a single Higgs doublet. In addition, high-scale validity of this scenario is not affected by the uncertainty in the strong coupling $\alpha_{s}\left(M_{Z}\right)$. The effect of a $\mathrm{CP}$-violating phase in the potential is also considered, it is found that one can find regions in the parameter space valid up to high scales for at least one illustrative value (viz. $\frac{\pi}{4}$ ) of the phase. The allowed regions of the parameter space, in terms of the various quartic couplings as well as the scalar mass eigenvalues are presented by us in detail, in the light of theoretical as well as collider bounds. The inclusion of $\mathbb{Z}_{2}$-breaking quartic couplings, too, is found to retain the high-scale validity of the theory over a large region.

Though the study is based on a type II $2 \mathrm{HDM}$, many of the results obtained here are expected to hold for a more general $2 \mathrm{HDM}$ as well. A situation where some departure can take place is, for example one where the Yukawa coupling of the bottom quark becomes comparable to, or more than, that of the top quark. One possibility to explore in such a case is to look for those regions where the large number of quartic couplings can rescue the scenario from an unstable vacuum. The results presented here are based on one-loop RG equations, in consonance with most similar studies in the context of 2HDM.

It should also be noted that we call those regions in the parameter space as allowed, where the vacuum is strictly stable. The inclusion of a metastable vacuum, with lifetime greater than the age of the universe, will lead to larger allowed regions.

On the whole, our conclusion is that it is possible to validate a $2 \mathrm{HDM}$ till scales as high as the Planck mass without any additional physics. While the issue of naturalness remains unaddressed in this statement, it is interesting to see that no current experimental measurement or theoretical restriction can affect high-scale validity, which is not the case for the single-doublet scenario.

\section{Acknowledgments}

We thank Shankha Banerjee, Joydeep Chakrabortty, Samrat Kadge and Sourov Roy for many helpful discussions. This work was partially supported by the funding available 
from the Department of Atomic Energy, Government of India, for the Regional Centre for Accelerator-based Particle Physics, Harish-Chandra Research Institute. Computational work for this study was partially carried out at the cluster computing facility in the HarishChandra Research Institute (http://cluster.hri.res.in).

\section{A Renormalization group (RG) equations}

The RG equations for the gauge couplings, for this model, are given by [9],

$$
\begin{aligned}
16 \pi^{2} \frac{d g_{s}}{d t} & =-7 g_{s}^{3}, \\
16 \pi^{2} \frac{d g}{d t} & =-3 g^{3}, \\
16 \pi^{2} \frac{d g^{\prime}}{d t} & =7 g^{\prime 3}
\end{aligned}
$$

Since we want to avoid $\mathrm{CP}$ violation coming from the quartic sector of the Higgs potential, we choose to keep $\lambda_{i}(i=1, \ldots, 7)$ real. In that case, the quartic couplings evolve according to,

$$
\begin{aligned}
16 \pi^{2} \frac{d \lambda_{1}}{d t}= & 12 \lambda_{1}^{2}+4 \lambda_{3}^{2}+4 \lambda_{3} \lambda_{4}+2 \lambda_{4}^{2}+2 \lambda_{5}^{2}+24 \lambda_{6}^{2}+\frac{3}{4}\left(3 g^{4}+g^{\prime 4}+2 g^{2} g^{\prime 2}\right) \\
& -\lambda_{1}\left(9 g^{2}+3 g^{\prime 2}-12 y_{b}^{2}-4 y_{\tau}^{2}\right)-12 y_{b}^{4}-4 y_{\tau}^{4} \\
16 \pi^{2} \frac{d \lambda_{2}}{d t}= & 12 \lambda_{2}^{2}+4 \lambda_{3}^{2}+4 \lambda_{3} \lambda_{4}+2 \lambda_{4}^{2}+2 \lambda_{5}^{2}+24 \lambda_{7}^{2} \\
& +\frac{3}{4}\left(3 g^{4}+g^{\prime 4}+2 g^{2} g^{\prime 2}\right)-3 \lambda_{2}\left(3 g^{2}+g^{\prime 2}-4 y_{t}^{2}\right)-12 y_{t}^{4} \\
16 \pi^{2} \frac{d \lambda_{3}}{d t}= & \left(\lambda_{1}+\lambda_{2}\right)\left(6 \lambda_{3}+2 \lambda_{4}\right)+4 \lambda_{3}^{2}+2 \lambda_{4}^{2}+2 \lambda_{5}^{2}+4\left(\lambda_{6}^{2}+\lambda_{7}^{2}\right) \\
& +16\left(\lambda_{6} \lambda_{7}\right)+\frac{3}{4}\left(3 g^{4}+g^{\prime 4}-2 g^{2} g^{\prime 2}\right) \\
& -\lambda_{3}\left(9 g^{2}+3 g^{\prime 2}-6 y_{t}^{2}-6 y_{b}^{2}-2 y_{\tau}^{2}\right)-12 y_{t}^{2} y_{b}^{2} \\
16 \pi^{2} \frac{d \lambda_{4}}{d t}= & 2\left(\lambda_{1}+\lambda_{2}\right) \lambda_{4}+8 \lambda_{3} \lambda_{4}+4 \lambda_{4}^{2}+8 \lambda_{5}^{2}+10\left(\lambda_{6}^{2}+\lambda_{7}^{2}\right)+4\left(\lambda_{6} \lambda_{7}\right) \\
& +3 g^{2} g^{\prime 2}-\lambda_{4}\left(9 g^{2}+3 g^{2}-6 y_{t}^{2}-6 y_{b}^{2}-2 y_{\tau}^{2}\right)+12 y_{t}^{2} y_{b}^{2} \\
16 \pi^{2} \frac{d \lambda_{5}}{d t}= & \left(2 \lambda_{1}+2 \lambda_{2}+8 \lambda_{3}+12 \lambda_{4}\right) \lambda_{5}+10\left(\lambda_{6}^{2}+\lambda_{7}^{2}\right)+4 \lambda_{6} \lambda_{7} \\
& -\lambda_{5}\left(9 g^{2}+3 g^{\prime 2}-6 y_{t}^{2}-6 y_{b}^{2}-2 y_{\tau}^{2}\right) \\
16 \pi^{2} \frac{d \lambda_{6}}{d t}= & \left(12 \lambda_{1}+6 \lambda_{3}+8 \lambda_{4}\right) \lambda_{6}+\left(6 \lambda_{3}+4 \lambda_{4}\right) \lambda_{7}+10 \lambda_{5} \lambda_{6}+2 \lambda_{5} \lambda_{7} \\
& -\lambda_{6}\left(9 g^{2}+3 g^{\prime 2}-9 y_{b}^{2}-3 y_{t}^{2}-3 y_{\tau}^{2}\right) \\
16 \pi^{2} \frac{d \lambda_{7}}{d t}= & \left(12 \lambda_{2}+6 \lambda_{3}+8 \lambda_{4}\right) \lambda_{7}+\left(6 \lambda_{3}+4 \lambda_{4}\right) \lambda_{6}+10 \lambda_{5} \lambda_{7}+2 \lambda_{5} \lambda_{6} \\
& -\lambda_{7}\left(9 g^{2}+3 g^{\prime 2}-9 y_{t}^{2}-3 y_{b}^{2}-y_{\tau}^{2}\right)
\end{aligned}
$$


For the Yukawa couplings the corresponding set of RG equations are,

$$
\begin{aligned}
16 \pi^{2} \frac{d y_{b}}{d t} & =y_{b}\left(-8 g_{s}^{2}-\frac{9}{4} g^{2}-\frac{5}{12} g^{\prime 2}+\frac{9}{2} y_{b}^{2}+y_{\tau}^{2}+\frac{1}{2} y_{t}^{2}\right) \\
16 \pi^{2} \frac{d y_{t}}{d t} & =y_{t}\left(-8 g_{s}^{2}-\frac{9}{4} g^{2}-\frac{17}{12} g^{\prime 2}+\frac{9}{2} y_{t}^{2}+\frac{1}{2} y_{b}^{2}\right) \\
16 \pi^{2} \frac{d y_{\tau}}{d t} & =y_{\tau}\left(-\frac{9}{4} g^{2}-\frac{15}{4} g^{\prime 2}+3 y_{b}^{2}+\frac{5}{2} y_{\tau}^{2}\right) .
\end{aligned}
$$

\section{B Unitarity bounds}

We perform a coupled channel analysis of $2 \rightarrow 2$ scattering involving fields in the scalar sector, to the leading order. The basis of neutral two-particle states is given by,

$$
\left\{w_{1}^{+} w_{2}^{-}, w_{2}^{+} w_{1}^{-}, h_{1} z_{2}, h_{2} z_{1}, z_{1} z_{2}, h_{1} h_{2}, h_{1} z_{1}, h_{2} z_{2}, w_{1}^{+} w_{1}^{-}, w_{2}^{+} w_{2}^{-}, \frac{z_{1} z_{1}}{\sqrt{2}}, \frac{z_{2} z_{2}}{\sqrt{2}}, \frac{h_{1} h_{1}}{\sqrt{2}}, \frac{h_{2} h_{2}}{\sqrt{2}}\right\}
$$

For the general $\lambda_{6}, \lambda_{7} \neq 0$ case, the $(14 \times 14)$ two-particle scattering matrix is given as follows:

$$
\mathcal{M}_{\mathrm{NC}}=\left(\begin{array}{ll}
\mathcal{A}_{7 \times 7} & \mathcal{B}_{7 \times 7} \\
\mathcal{B}_{7 \times 7}^{\dagger} & \mathcal{C}_{7 \times 7}
\end{array}\right),
$$

where $\mathcal{A}, \mathcal{B}$ and $\mathcal{C}$ are given by,

$$
\begin{aligned}
& \mathcal{A}_{7 \times 7}=\left(\begin{array}{ccccccc}
\lambda_{3}+\lambda_{4} & 2 \lambda_{5} & \frac{i}{2}\left(\lambda_{4}-\lambda_{5}\right) & \frac{i}{2}\left(-\lambda_{4}+\lambda_{5}\right) & \frac{1}{2}\left(\lambda_{4}+\lambda_{5}\right) & \frac{1}{2}\left(\lambda_{4}+\lambda_{5}\right) & 0 \\
2 \lambda_{5} & \lambda_{3}+\lambda_{4} & \frac{i}{2}\left(-\lambda_{4}+\lambda_{5}\right) & \frac{i}{2}\left(\lambda_{4}-\lambda_{5}\right) & \frac{1}{2}\left(\lambda_{4}+\lambda_{5}\right) & \frac{1}{2}\left(\lambda_{4}+\lambda_{5}\right) & 0 \\
\frac{i}{2}\left(-\lambda_{4}+\lambda_{5}\right) & \frac{i}{2}\left(\lambda_{4}-\lambda_{5}\right) & \left(\lambda_{3}+\lambda_{4}-\lambda_{5}\right) & \lambda_{5} & 0 & 0 & \lambda_{6} \\
\frac{i}{2}\left(\lambda_{4}-\lambda_{5}\right) & \frac{i}{2}\left(-\lambda_{4}+\lambda_{5}\right) & \lambda_{5} & \left(\lambda_{3}+\lambda_{4}-\lambda_{5}\right) & 0 & 0 & \lambda_{6} \\
\frac{1}{2}\left(\lambda_{4}+\lambda_{5}\right) & \frac{1}{2}\left(\lambda_{4}+\lambda_{5}\right) & 0 & 0 & \left(\lambda_{3}+\lambda_{4}+\lambda_{5}\right) & \lambda_{5} & 0 \\
\frac{1}{2}\left(\lambda_{4}+\lambda_{5}\right) & \frac{1}{2}\left(\lambda_{4}+\lambda_{5}\right) & 0 & 0 & \lambda_{5} & \left(\lambda_{3}+\lambda_{4}+\lambda_{5}\right) & 0 \\
0 & 0 & \lambda_{6} & \lambda_{6} & 0 & 0 & \lambda_{1}
\end{array}\right), \\
& \mathcal{B}_{7 \times 7}=\left(\begin{array}{ccccccc}
0 & 2 \lambda_{6} & 2 \lambda_{7} & \frac{\lambda_{6}}{\sqrt{2}} & \frac{\lambda_{7}}{\sqrt{2}} & \frac{\lambda_{6}}{\sqrt{2}} & \frac{\lambda_{7}}{\sqrt{2}} \\
0 & 2 \lambda_{6} & 2 \lambda_{7} & \frac{\lambda_{6}}{\sqrt{2}} & \frac{\lambda_{7}}{\sqrt{2}} & \frac{\lambda_{6}}{\sqrt{2}} & \frac{\lambda_{7}}{\sqrt{2}} \\
\lambda_{7} & 0 & 0 & 0 & 0 & 0 & 0 \\
\lambda_{7} & 0 & 0 & 0 & 0 & 0 & 0 \\
0 & \lambda_{6} & \lambda_{7} & \frac{3 \lambda_{6}}{\sqrt{2}} & \frac{3 \lambda_{7}}{\sqrt{2}} & \frac{\lambda_{6}}{\sqrt{2}} & \frac{\lambda_{7}}{\sqrt{2}} \\
0 & \lambda_{6} & \lambda_{7} & \frac{\lambda_{6}}{\sqrt{2}} & \frac{\lambda_{7}}{\sqrt{2}} & \frac{3 \lambda_{6}}{\sqrt{2}} & \frac{3 \lambda_{7}}{\sqrt{2}} \\
\lambda_{5} & 0 & 0 & 0 & 0 & 0 & 0
\end{array}\right) \\
& \mathcal{C}_{7 \times 7}=\left(\begin{array}{ccccccc}
\lambda_{2} & 0 & 0 & 0 & 0 & 0 & 0 \\
0 & 2 \lambda_{1} & \left(\lambda_{3}+\lambda_{4}\right) & \frac{\lambda_{1}}{\sqrt{2}} & \frac{\lambda_{3}}{\sqrt{2}} & \frac{\lambda_{1}}{\sqrt{2}} & \frac{\lambda_{1}}{\sqrt{2}} \\
0 & \left(\lambda_{3}+\lambda_{4}\right) & 2 \lambda_{2} & \frac{\lambda_{3}}{\sqrt{2}} & \frac{\lambda_{2}}{\sqrt{2}} & \frac{\lambda_{3}}{\sqrt{2}} & \frac{\lambda_{2}}{\sqrt{2}} \\
0 & \frac{\lambda_{1}}{\sqrt{2}} & \frac{\lambda_{3}}{\sqrt{2}} & \frac{3 \lambda_{1}}{2} & \frac{1}{2}\left(\lambda_{3}+\lambda_{4}+\lambda_{5}\right) & \frac{\lambda_{1}}{2} & \frac{1}{2}\left(\lambda_{3}+\lambda_{4}-\lambda_{5}\right) \\
0 & \frac{\lambda_{3}}{\sqrt{2}} & \frac{\lambda_{2}}{\sqrt{2}} & \frac{1}{2}\left(\lambda_{3}+\lambda_{4}+\lambda_{5}\right) & \frac{3 \lambda_{2}}{2} & \frac{1}{2}\left(\lambda_{3}+\lambda_{4}-\lambda_{5}\right) & \frac{\lambda_{2}}{2} \\
0 & \frac{\lambda_{1}}{\sqrt{2}} & \frac{\lambda_{3}}{\sqrt{2}} & \frac{\lambda_{1}}{2} & \frac{1}{2}\left(\lambda_{3}+\lambda_{4}-\lambda_{5}\right) & \frac{3 \lambda_{1}}{2} & \frac{1}{2}\left(\lambda_{3}+\lambda_{4}+\lambda_{5}\right) \\
0 & \frac{\lambda_{3}}{\sqrt{2}} & \frac{\lambda_{2}}{\sqrt{2}} & \frac{1}{2}\left(\lambda_{3}+\lambda_{4}-\lambda_{5}\right) & \frac{\lambda_{2}}{2} & \frac{1}{2}\left(\lambda_{3}+\lambda_{4}+\lambda_{5}\right) & \frac{3 \lambda_{2}}{2}
\end{array}\right) .
\end{aligned}
$$


The constraint imposed by unitarity is then given by $\left|a_{i}\right| \leq 8 \pi$, where $a_{i}(i=1, \ldots, 14)$ are eigenvalues of the matrix $\mathcal{M}$. The eigenvalues of $\mathcal{M}$ are evaluated numerically in the present study. However, in the absence of hard $\mathbb{Z}_{2}$ breaking, i.e., when $\lambda_{6}, \lambda_{7}=0$, the matrix decomposes into blocks and analytical expressions for its eigenvalues can be obtained in simple forms which are listed below.

$$
\begin{aligned}
a_{ \pm} & =\frac{3}{2}\left(\lambda_{1}+\lambda_{2}\right) \pm \sqrt{\frac{9}{4}\left(\lambda_{1}-\lambda_{2}\right)^{2}+\left(2 \lambda_{3}+\lambda_{4}\right)^{2}}, \\
b_{ \pm} & =\frac{1}{2}\left(\lambda_{1}+\lambda_{2}\right) \pm \sqrt{\frac{1}{4}\left(\lambda_{1}-\lambda_{2}\right)^{2}+\lambda_{4}^{2}}, \\
c_{ \pm} & =d_{ \pm}=\frac{1}{2}\left(\lambda_{1}+\lambda_{2}\right) \pm \sqrt{\frac{1}{4}\left(\lambda_{1}-\lambda_{2}\right)^{2}+\lambda_{5}^{2}}, \\
e_{1} & =\left(\lambda_{3}+2 \lambda_{4}-3 \lambda_{5}\right), \\
e_{2} & =\left(\lambda_{3}-\lambda_{5}\right), \\
f_{1} & =f_{2}=\left(\lambda_{3}+\lambda_{4}\right), \\
f_{+} & =\left(\lambda_{3}+2 \lambda_{4}+3 \lambda_{5}\right), \\
f_{-} & =\left(\lambda_{3}+\lambda_{5}\right) .
\end{aligned}
$$

The matrix corresponding to the overall singly charged states,

$$
\left\{h_{1} w_{1}^{+}, h_{2} w_{1}^{+}, z_{1} w_{1}^{+}, z_{2} w_{1}^{+}, h_{1} w_{2}^{+}, h_{2} w_{2}^{+}, z_{1} w_{2}^{+}, z_{2} w_{2}^{+}\right\}
$$

is given by,

$$
\mathcal{M}_{\mathrm{CC}}=\left(\begin{array}{cccccccc}
\lambda_{1} & \lambda_{6} & 0 & 0 & \lambda_{6} & \frac{1}{2}\left(\lambda_{4}+\lambda_{5}\right) & 0 & \frac{i\left(\lambda_{4}-\lambda_{5}\right)}{2} \\
\lambda_{6} & \lambda_{3} & 0 & 0 & \frac{\lambda_{4}+\lambda_{5}}{2} & \lambda_{7} & \frac{-i\left(\lambda_{4}-\lambda_{5}\right)}{2} & 0 \\
0 & 0 & \lambda_{1} & \lambda_{6} & 0 & \frac{-i\left(\lambda_{4}-\lambda_{5}\right)}{2} & \lambda_{6} & \frac{\lambda_{4}+\lambda_{5}}{2} \\
0 & 0 & \lambda_{6} & \lambda_{3} & \frac{i\left(\lambda_{4}-\lambda_{5}\right)}{2} & 0 & \frac{\lambda_{4}+\lambda_{5}}{2} & \lambda_{7} \\
\lambda_{6} & \frac{\lambda_{4}+\lambda_{5}}{2} & 0 & \frac{-i\left(\lambda_{4}-\lambda_{5}\right)}{2} & \lambda_{3} & \lambda_{7} & 0 & 0 \\
\frac{\lambda_{4}+\lambda_{5}}{2} & \lambda_{7} & \frac{i\left(\lambda_{4}-\lambda_{5}\right)}{2} & 0 & \lambda_{7} & \lambda_{2} & 0 & 0 \\
0 & \frac{i\left(\lambda_{4}-\lambda_{5}\right)}{2} & \lambda_{6} & \frac{\lambda_{4}+\lambda_{5}}{2} & 0 & 0 & \lambda_{3} & \lambda_{7} \\
\frac{-i\left(\lambda_{4}-\lambda_{5}\right)}{2} & 0 & \frac{\lambda_{4}+\lambda_{5}}{2} & \lambda_{7} & 0 & 0 & \lambda_{7} & \lambda_{2}
\end{array}\right) .
$$

Again for the case $\lambda_{6}, \lambda_{7}=0$, the eigenvalues of $\mathcal{M}_{\mathrm{CC}}$ are, $b_{ \pm}, c_{ \pm}, e_{2}, f_{1}, f_{-}$and $p=\left(\lambda_{3}-\lambda_{4}\right)$. 
Open Access. This article is distributed under the terms of the Creative Commons Attribution License (CC-BY 4.0), which permits any use, distribution and reproduction in any medium, provided the original author(s) and source are credited.

\section{References}

[1] ATLAS collaboration, Observation of a new particle in the search for the Standard Model Higgs boson with the ATLAS detector at the LHC, Phys. Lett. B 716 (2012) 1 [arXiv: 1207.7214] [INSPIRE].

[2] CMS collaboration, Observation of a new boson at a mass of $125 \mathrm{GeV}$ with the CMS experiment at the LHC, Phys. Lett. B 716 (2012) 30 [arXiv:1207.7235] [INSPIRE].

[3] ATLAS collaboration, Evidence for the spin-0 nature of the Higgs boson using ATLAS data, Phys. Lett. B 726 (2013) 120 [arXiv:1307.1432] [INSPIRE].

[4] ATLAS collaboration, Measurements of the properties of the Higgs-like boson in the two photon decay channel with the ATLAS detector using $25 \mathrm{fb}^{-1}$ of proton-proton collision data, ATLAS-CONF-2013-012 [ATLAS-COM-CONF-2013-015] (2013).

[5] A. Freitas and P. Schwaller, Higgs CP Properties From Early LHC Data, Phys. Rev. D 87 (2013) 055014 [arXiv: 1211.1980] [INSPIRE].

[6] A. Djouadi, R.M. Godbole, B. Mellado and K. Mohan, Probing the spin-parity of the Higgs boson via jet kinematics in vector boson fusion, Phys. Lett. B 723 (2013) 307 [arXiv: 1301.4965] [INSPIRE].

[7] A. Djouadi and G. Moreau, The couplings of the Higgs boson and its CP properties from fits of the signal strengths and their ratios at the $7+8 \mathrm{TeV}$ LHC, Eur. Phys. J. C 73 (2013) 2512 [arXiv: 1303.6591] [INSPIRE].

[8] J.F. Gunion, H.E. Haber, G.L. Kane and S. Dawson, The Higgs Hunter's Guide, Front. Phys. 80 (2000) 1 [INSPIRE].

[9] G.C. Branco et al., Theory and phenomenology of two-Higgs-doublet models, Phys. Rept. 516 (2012) 1 [arXiv: 1106.0034] [INSPIRE].

[10] N.G. Deshpande and E. Ma, Comment on Weinberg's Gauge Theory of CP Nonconservation, Phys. Rev. D 16 (1977) 1583 [INSPIRE].

[11] R.N. Mohapatra and G. Senjanović, Neutrino Mass and Spontaneous Parity Violation, Phys. Rev. Lett. 44 (1980) 912 [INSPIRE].

[12] M. Holthausen, K.S. Lim and M. Lindner, Planck scale Boundary Conditions and the Higgs Mass, JHEP 02 (2012) 037 [arXiv:1112.2415] [INSPIRE].

[13] J. Elias-Miro et al., Higgs mass implications on the stability of the electroweak vacuum, Phys. Lett. B 709 (2012) 222 [arXiv:1112.3022] [INSPIRE].

[14] G. Degrassi et al., Higgs mass and vacuum stability in the Standard Model at NNLO, JHEP 08 (2012) 098 [arXiv: 1205.6497] [INSPIRE].

[15] M.F. Zoller, Vacuum stability in the SM and the three-loop $\beta$-function for the Higgs self-interaction, arXiv:1209.5609 [INSPIRE].

[16] D. Buttazzo et al., Investigating the near-criticality of the Higgs boson, JHEP 12 (2013) 089 [arXiv: 1307.3536] [INSPIRE]. 
[17] S. Davidson and H.E. Haber, Basis-independent methods for the two-Higgs-doublet model, Phys. Rev. D 72 (2005) 035004 [Erratum ibid. D 72 (2005) 099902] [hep-ph/0504050] [INSPIRE].

[18] H.E. Haber and D. O'Neil, Basis-independent methods for the two-Higgs-doublet model. II. The Significance of tan beta, Phys. Rev. D 74 (2006) 015018 [hep-ph/0602242] [INSPIRE].

[19] H.E. Haber and D. O'Neil, Basis-independent methods for the two-Higgs-doublet model III: The CP-conserving limit, custodial symmetry and the oblique parameters S, T, U, Phys. Rev. D 83 (2011) 055017 [arXiv: 1011.6188] [INSPIRE].

[20] A. Barroso, P.M. Ferreira, I.P. Ivanov and R. Santos, Metastability bounds on the two Higgs doublet model, JHEP 06 (2013) 045 [arXiv: 1303.5098] [INSPIRE].

[21] B. Gorczyca and M. Krawczyk, Tree-Level Unitarity Constraints for the SM-like 2HDM, arXiv:1112.5086 [INSPIRE].

[22] K. Kannike, Vacuum Stability Conditions From Copositivity Criteria, Eur. Phys. J. C 72 (2012) 2093 [arXiv: 1205.3781] [inSPIRE].

[23] J. Kopp and M. Nardecchia, Flavor and CP-violation in Higgs decays, JHEP 10 (2014) 156 [arXiv: 1406.5303] [INSPIRE].

[24] S. Inoue, M.J. Ramsey-Musolf and Y. Zhang, CPV Phenomenology of Flavor Conserving Two Higgs Doublet Models, Phys. Rev. D 89 (2014) 115023 [arXiv:1403.4257] [InSPIRE].

[25] A. Arhrib, E. Christova, H. Eberl and E. Ginina, CP violation in charged Higgs production and decays in the Complex Two Higgs Doublet Model, JHEP 04 (2011) 089 [arXiv: 1011.6560] [INSPIRE].

[26] P. Osland, A. Pukhov, G.M. Pruna and M. Purmohammadi, Phenomenology of charged scalars in the CP-Violating Inert-Doublet Model, JHEP 04 (2013) 040 [arXiv:1302.3713] [INSPIRE].

[27] S.L. Glashow and S. Weinberg, Natural Conservation Laws for Neutral Currents, Phys. Rev. D 15 (1977) 1958 [INSPIRE].

[28] E.A. Paschos, Diagonal Neutral Currents, Phys. Rev. D 15 (1977) 1966 [inSPIRE].

[29] A. Arhrib, Top and Higgs flavor changing neutral couplings in two Higgs doublets model, Phys. Rev. D 72 (2005) 075016 [hep-ph/0510107] [INSPIRE].

[30] A. Dery, A. Efrati, G. Hiller, Y. Hochberg and Y. Nir, Higgs couplings to fermions: 2HDM with MFV, JHEP 08 (2013) 006 [arXiv: 1304.6727] [INSPIRE].

[31] G. Cvetič, S.S. Hwang and C.S. Kim, One loop renormalization group equations of the general framework with two Higgs doublets, Int. J. Mod. Phys. A 14 (1999) 769 [hep-ph/9706323] [INSPIRE].

[32] G. Cvetič, C.S. Kim and S.S. Hwang, Higgs mediated flavor changing neutral currents in the general framework with two Higgs doublets: An RGE analysis, Phys. Rev. D 58 (1998) 116003 [hep-ph/9806282] [INSPIRE].

[33] A. Pich and P. Tuzon, Yukawa Alignment in the Two-Higgs-Doublet Model, Phys. Rev. D 80 (2009) 091702 [arXiv:0908.1554] [INSPIRE].

[34] J.F. Gunion and H.E. Haber, The CP conserving two Higgs doublet model: The approach to the decoupling limit, Phys. Rev. D 67 (2003) 075019 [hep-ph/0207010] [INSPIRE]. 
[35] B.W. Lee, C. Quigg and H.B. Thacker, Weak Interactions at Very High-Energies: The Role of the Higgs Boson Mass, Phys. Rev. D 16 (1977) 1519 [InSPIRE].

[36] I.F. Ginzburg and I.P. Ivanov, Tree-level unitarity constraints in the most general 2HDM, Phys. Rev. D 72 (2005) 115010 [hep-ph/0508020] [InSPIRE].

[37] G. Bhattacharyya, D. Das, P.B. Pal and M.N. Rebelo, Scalar sector properties of two-Higgs-doublet models with a global U(1) symmetry, JHEP 10 (2013) 081 [arXiv: 1308.4297] [INSPIRE].

[38] B. Grzadkowski, O.M. Ogreid and P. Osland, Diagnosing CP properties of the 2HDM, JHEP 01 (2014) 105 [arXiv: 1309.6229] [INSPIRE].

[39] L. Basso et al., The CP-violating type-II 2HDM and Charged Higgs boson benchmarks, PoS (Corfu2012) 029 [arXiv: 1305.3219] [INSPIRE].

[40] S. Kanemura, T. Kubota and E. Takasugi, Lee-Quigg-Thacker bounds for Higgs boson masses in a two doublet model, Phys. Lett. B 313 (1993) 155 [hep-ph/9303263] [INSPIRE].

[41] A.G. Akeroyd, A. Arhrib and E.-M. Naimi, Note on tree level unitarity in the general two Higgs doublet model, Phys. Lett. B 490 (2000) 119 [hep-ph/0006035] [INSPIRE].

[42] J. Horejsi and M. Kladiva, Tree-unitarity bounds for THDM Higgs masses revisited, Eur. Phys. J. C 46 (2006) 81 [hep-ph/0510154] [INSPIRE].

[43] M. Sher, Electroweak Higgs Potentials and Vacuum Stability, Phys. Rept. 179 (1989) 273 [INSPIRE].

[44] S. Nie and M. Sher, Vacuum stability bounds in the two Higgs doublet model, Phys. Lett. B 449 (1999) 89 [hep-ph/9811234] [INSPIRE].

[45] P.M. Ferreira and D.R.T. Jones, Bounds on scalar masses in two Higgs doublet models, JHEP 08 (2009) 069 [arXiv:0903.2856] [INSPIRE].

[46] P.M. Ferreira, R. Santos and A. Barroso, Stability of the tree-level vacuum in two Higgs doublet models against charge or CP spontaneous violation, Phys. Lett. B 603 (2004) 219 [Erratum ibid. B 629 (2005) 114] [hep-ph/0406231] [InSPIRE].

[47] J. Bijnens, J. Lu and J. Rathsman, Constraining General 2HDM by the Evolution of Yukawa Couplings, PoS (CHARGED 2012) 023 [arXiv: 1301.7451] [INSPIRE].

[48] I. Chakraborty and A. Kundu, Two-Higgs doublet models confront the naturalness problem, arXiv: 1404.3038 [INSPIRE].

[49] ATLAS collaboration, Measurement of the Higgs boson mass from the $H \rightarrow \gamma \gamma$ and $H \rightarrow Z Z^{*} \rightarrow 4 \ell$ channels with the ATLAS detector using $25 \mathrm{fb}^{-1}$ of pp collision data, Phys. Rev. D 90 (2014) 052004 [arXiv: 1406.3827] [INSPIRE].

[50] F. Mahmoudi and O. Stal, Flavor constraints on the two-Higgs-doublet model with general Yukawa couplings, Phys. Rev. D 81 (2010) 035016 [arXiv:0907.1791] [INSPIRE].

[51] O. Deschamps et al., The Two Higgs Doublet of Type II facing flavour physics data, Phys. Rev. D 82 (2010) 073012 [arXiv:0907.5135] [INSPIRE].

[52] Particle Data Group collaboration, J. Beringer et al., Review of Particle Physics (RPP), Phys. Rev. D 86 (2012) 010001 [InSPIRE].

[53] M.E. Peskin and T. Takeuchi, Estimation of oblique electroweak corrections, Phys. Rev. D 46 (1992) 381 [INSPIRE]. 
[54] H.-J. He, N. Polonsky and S.-f. Su, Extra families, Higgs spectrum and oblique corrections, Phys. Rev. D 64 (2001) 053004 [hep-ph/0102144] [InSPIRE].

[55] W. Grimus, L. Lavoura, O.M. Ogreid and P. Osland, The oblique parameters in multi-Higgs-doublet models, Nucl. Phys. B 801 (2008) 81 [arXiv:0802.4353] [INSPIRE].

[56] G. Funk, D. O'Neil and R.M. Winters, What the Oblique Parameters S, T and U and Their Extensions Reveal About the 2HDM: A Numerical Analysis, Int. J. Mod. Phys. A 27 (2012) 1250021 [arXiv: 1110.3812] [INSPIRE].

[57] GFitter Group collaboration, M. Baak et al., The global electroweak fit at NNLO and prospects for the LHC and ILC, Eur. Phys. J. C 74 (2014) 3046 [arXiv:1407.3792] [INSPIRE].

[58] ATLAS collaboration, Search for the Standard Model Higgs boson in $H \rightarrow \tau^{+} \tau$ - decays in proton-proton collisions with the ATLAS detector, ATLAS-CONF-2012-160

[ATLAS-COM-CONF-2012-196] (2012).

[59] ATLAS collaboration, Search for the bb decay of the Standard Model Higgs boson in associated W/ZH production with the ATLAS detector, ATLAS-CONF-2013-079 [ATLAS-COM-CONF-2013-080] (2013).

[60] ATLAS collaboration, Measurements of Higgs boson production and couplings in diboson final states with the ATLAS detector at the LHC, Phys. Lett. B 726 (2013) 88 [arXiv: 1307.1427] [INSPIRE].

[61] CMS collaboration, Precise determination of the mass of the Higgs boson and studies of the compatibility of its couplings with the standard model, CMS-PAS-HIG-14-009.

[62] B. Coleppa, F. Kling and S. Su, Constraining Type II 2HDM in Light of LHC Higgs Searches, JHEP 01 (2014) 161 [arXiv:1305.0002] [INSPIRE].

[63] P.M. Ferreira, R. Santos, M. Sher and J.P. Silva, Implications of the LHC two-photon signal for two-Higgs-doublet models, Phys. Rev. D 85 (2012) 077703 [arXiv:1112.3277] [INSPIRE].

[64] P.M. Ferreira, R. Santos, M. Sher and J.P. Silva, 2HDM confronting LHC data, arXiv: 1305.4587 [INSPIRE].

[65] A. Drozd, B. Grzadkowski, J.F. Gunion and Y. Jiang, Two-Higgs-Doublet Models and Enhanced Rates for a $125 \mathrm{GeV}$ Higgs, JHEP 05 (2013) 072 [arXiv:1211.3580] [INSPIRE].

[66] H.S. Cheon and S.K. Kang, Constraining parameter space in type-II two-Higgs doublet model in light of a $126 \mathrm{GeV}$ Higgs boson, JHEP 09 (2013) 085 [arXiv:1207.1083] [INSPIRE].

[67] A. Drozd, B. Grzadkowski, J.F. Gunion and Y. Jiang, 2HDM and Enhanced Rates in $\gamma \gamma$ Channel, Acta Phys. Polon. B 44 (2013) 1417 [InSPIRE].

[68] M. Krawczyk, D. Sokolowska and B. Swiezewska, 2HDM with $Z_{2}$ symmetry in light of new LHC data, J. Phys. Conf. Ser. 447 (2013) 012050 [arXiv:1303.7102] [InSPIRE].

[69] L. Wang and X.-F. Han, Status of the aligned two-Higgs-doublet model confronted with the Higgs data, JHEP 04 (2014) 128 [arXiv:1312.4759] [INSPIRE].

[70] B. Dumont, J.F. Gunion, Y. Jiang and S. Kraml, Constraints on and future prospects for Two-Higgs-Doublet Models in light of the LHC Higgs signal, Phys. Rev. D 90 (2014) 035021 [arXiv: 1405.3584] [INSPIRE].

[71] A. Celis, V. Ilisie and A. Pich, LHC constraints on two-Higgs doublet models, JHEP 07 (2013) 053 [arXiv:1302.4022] [INSPIRE]. 
[72] A. Celis, V. Ilisie and A. Pich, Towards a general analysis of LHC data within two-Higgs-doublet models, JHEP 12 (2013) 095 [arXiv: 1310.7941] [INSPIRE].

[73] O. Eberhardt, U. Nierste and M. Wiebusch, Status of the two-Higgs-doublet model of type-II, JHEP 07 (2013) 118 [arXiv: 1305.1649] [INSPIRE].

[74] S. Kanemura, K. Tsumura, K. Yagyu and H. Yokoya, Fingerprinting nonminimal Higgs sectors, Phys. Rev. D 90 (2014) 075001 [arXiv: 1406.3294] [InSPIRE].

[75] S. Banerjee, S. Mukhopadhyay and B. Mukhopadhyaya, New Higgs interactions and recent data from the LHC and the Tevatron, JHEP 10 (2012) 062 [arXiv: 1207.3588] [INSPIRE].

[76] T. Corbett, O.J.P. Eboli, J. Gonzalez-Fraile and M.C. Gonzalez-Garcia, Constraining anomalous Higgs interactions, Phys. Rev. D 86 (2012) 075013 [arXiv:1207.1344] [InSPIRE].

[77] J.R. Espinosa, C. Grojean, M. Muhlleitner and M. Trott, Fingerprinting Higgs Suspects at the LHC, JHEP 05 (2012) 097 [arXiv: 1202.3697] [InSPIRE].

[78] P.P. Giardino, K. Kannike, M. Raidal and A. Strumia, Reconstructing Higgs boson properties from the LHC and Tevatron data, JHEP 06 (2012) 117 [arXiv:1203.4254] [INSPIRE].

[79] M. Klute, R. Lafaye, T. Plehn, M. Rauch and D. Zerwas, Measuring Higgs Couplings from LHC Data, Phys. Rev. Lett. 109 (2012) 101801 [arXiv:1205.2699] [INSPIRE].

[80] A. Azatov et al., Determining Higgs couplings with a model-independent analysis of $h \rightarrow \gamma \gamma$, JHEP 06 (2012) 134 [arXiv: 1204.4817] [INSPIRE].

[81] D. Eriksson, J. Rathsman and O. Stal, 2HDMC: Two-Higgs-Doublet Model Calculator Physics and Manual, Comput. Phys. Commun. 181 (2010) 189 [arXiv:0902.0851] [InSPIRE].

[82] S. Moch, U. Langenfeld and P. Uwer, The Top-Quark's Running Mass, PoS (RADCOR2009) 030 [arXiv: 1001.3987] [INSPIRE].

[83] U. Langenfeld, S. Moch and P. Uwer, Measuring the running top-quark mass, Nuovo Cim. C033N4 (2010) 47.

[84] D0 collaboration, V.M. Abazov et al., Precision measurement of the top-quark mass in lepton+jets final states, Phys. Rev. Lett. 113 (2014) 032002 [arXiv:1405.1756] [INSPIRE].

[85] L. Basso et al., Probing the charged Higgs boson at the LHC in the CP-violating type-II 2HDM, JHEP 11 (2012) 011 [arXiv:1205.6569] [INSPIRE]. 